Louisiana State University

LSU Digital Commons

Faculty Publications

Department of Biological Sciences

4-1-2016

\title{
The extrinsic proteins of photosystem II: update
}

Johnna L. Roose

Louisiana State University

Laurie K. Frankel

Louisiana State University

Manjula P. Mummadisetti

Louisiana State University

Terry M. Bricker

Louisiana State University

Follow this and additional works at: https://digitalcommons.Isu.edu/biosci_pubs

\section{Recommended Citation}

Roose, J., Frankel, L., Mummadisetti, M., \& Bricker, T. (2016). The extrinsic proteins of photosystem II: update. Planta, 243 (4), 889-908. https://doi.org/10.1007/s00425-015-2462-6

This Article is brought to you for free and open access by the Department of Biological Sciences at LSU Digital Commons. It has been accepted for inclusion in Faculty Publications by an authorized administrator of LSU Digital Commons. For more information, please contact ir@lsu.edu. 


\title{
The extrinsic proteins of photosystem II: update
}

\author{
Johnna L. Roose ${ }^{1}$ Laurie K. Frankel ${ }^{1}$ Manjula P. Mummadisetti ${ }^{1}$. \\ Terry M. Bricker ${ }^{1}$
}

Received: 11 November 2015 / Accepted: 25 December 2015/Published online: 12 January 2016

(C) Springer-Verlag Berlin Heidelberg 2016

\begin{abstract}
Main conclusion Recent investigations have provided important new insights into the structures and functions of the extrinsic proteins of Photosystem II.
\end{abstract}

This review is an update of the last major review on the extrinsic proteins of Photosystem II (Bricker et al., Biochemistry 31:4623-4628 2012). In this report, we will examine advances in our understanding of the structure and function of these components. These proteins include $\mathrm{PsbO}$, which is uniformly present in all oxygenic organisms, the PsbU, PsbV, CyanoQ, and CyanoP proteins, found in the cyanobacteria, and the PsbP, PsbQ and PsbR proteins, found in the green plant lineage. These proteins serve to stabilize the $\mathrm{Mn}_{4} \mathrm{CaO}_{5}$ cluster and optimize oxygen evolution at physiological calcium and chloride concentrations. The mechanisms used to perform these functions, however, remain poorly understood. Recently, important new findings have significantly advanced our understanding of the structures, locations and functions of these important subunits. We will discuss the biochemical, structural and genetic studies that have been used to elucidate the roles played by these proteins within the photosystem and their locations within the photosynthetic complex. Additionally, we will examine open questions needing to be addressed to provide a coherent picture of the role of these components within the photosystem.

Terry M. Bricker

btbric@1su.edu

1 Division of Biochemistry and Molecular Biology, Department of Biological Sciences, Louisiana State University, Baton Rouge, LA 70803, USA
Keywords PsbO - PsbU - PsbV - CyanoQ - CyanoP . PsbP · PsbQ · PsbR

\section{Introduction}

Photosystem II (PS II) is a light energy-driven waterplastoquinone oxidoreductase. In all known oxygenic organisms light energy is trapped by light-harvesting pigment arrays and transferred to the reaction center of PS II. Primary charge separation (see (Cardona et al. 2012), for an authoritative review) occurs between $\mathrm{Chl}_{\mathrm{D} 1}$ and $\mathrm{Pheo}_{\mathrm{D} 1}$, yielding $\mathrm{Ch}_{\mathrm{D} 1}^{+} \mathrm{Pheo}_{\mathrm{D} 1}^{-}$. This charge separation is stabilized by rapid oxidation of $\mathrm{P}_{\mathrm{D} 1}$, and by sequential electron transfer from $\mathrm{Pheo}-\overline{\mathrm{D} 1}$ to protein-bound plastoquinones, first to $\mathrm{Q}_{\mathrm{A}}$ and then to $\mathrm{Q}_{\mathrm{B}}$. The accumulation of two reducing equivalents on $\mathrm{Q}_{\mathrm{B}}$ leads to its protonation and the formation of plastoquinol, which is then released from PS II. $\mathrm{P}_{\mathrm{D} 1}^{+}$ is reduced by $\mathrm{Y}_{\mathrm{Z}}$, the residue $\mathrm{D} 1:{ }^{161} \mathrm{Y}$, forming $\mathrm{Y}_{\mathrm{Z}}$. The oxidation of $Y_{Z}$ leads to the release of a proton to a proton transfer pathway, which ultimately leads to its release to the lumen. The subsequent reduction of $\mathrm{Y}_{\mathrm{Z}}$ by protoncoupled electron transport leads to the accumulation of an oxidizing equivalent in the $\mathrm{Mn}_{4} \mathrm{CaO}_{5}$ cluster that forms the oxygen-evolving active site of the photosystem. The accumulation of four oxidizing equivalents in this metal cluster and/or in its ligands leads to the release of dioxygen from the complex, with the subsequent binding of two new substrate water molecules [for recent reviews, see (Vinyard et al. 2013; Yano and Yachandra 2014)].

The core intrinsic protein complex of PS II contains at least seventeen subunits (Umena et al. 2011). In higher plants and cyanobacteria seven of these subunits appear to be absolutely required for oxygen evolution and photoautotrophic growth, including PsbA (D1) and PsbD (D2), 
PsbB (CP47) and PsbC (CP43), the PsbE and PsbF subunits of cytochrome $b_{559}$, and PsbL. Genetic deletion of these components leads to loss of PS II assembly and the ability to evolve oxygen. The other ten subunits are low molecular mass intrinsic components and their functions remain obscure; in most instances their genetic deletion only modestly affects PS II assembly and function. PS II complexes containing only these intrinsic subunits can evolve oxygen, however, they do so at low rates, and require high, non-physiological levels of calcium and chloride (Bricker 1992). Optimal oxygen evolution capability requires a portfolio of extrinsic subunits. These include PsbO, which is found in all oxygenic organisms, and either PsbU, PsbV, CyanoQ and CyanoP, which are found in cyanobacteria, or PsbP, PsbQ and PsbR, which are found in the green plant lineage. Since the last major review article (Bricker et al. 2012) examining the extrinsic proteins of PS II, significant advances have been made concerning the structure and function of these components within the photosystem. The purpose of this communication is to provide a short, contemporary update examining these recent studies and how these integrate into our overall understanding of these important protein components of the photosystem. The reader should consult recent comprehensive reviews for an overview of earlier work and for in-depth examination of the structures and functions of these PS II components [for instance, see (Bricker and Burnap 2005; Nelson and Yocum 2006; Roose et al. 2007a; Suorsa and Aro 2007; Ifuku et al. 2008; Bricker et al. 2012, 2013; Ifuku 2014)].

\section{PsbO}

Functionally, the PsbO protein stabilizes the $\mathrm{Mn}_{4} \mathrm{CaO}_{5}$ cluster to sub-optimal chloride concentrations and protects the cluster from exogenous reductants (Bricker and Frankel 1998; Bricker et al. 2012). It is believed to play these roles in all oxygenic organisms. It may also assist in maintaining (along with PsbP and PsbQ) the association of calcium with the active site (Bricker and Frankel 2008) (however, see (Allahverdiyeva et al. 2009) for a different viewpoint). The mechanism(s) by which it accomplishes these processes, however, remains very unclear.

The extrinsic proteins on the lumenal side of PS II, as well as the extensive lumenal domains of the intrinsic proteins PsbC, PsbB, PsbA and PsbD, collectively maintain the proper environment for water oxidation (Bricker et al. 2012). Yet, water is a substrate and must gain access to this buried active site. Likewise, oxygen and $\mathrm{H}^{+}$produced at the $\mathrm{Mn}_{4} \mathrm{CaO}_{5}$ cluster must also exit the enzyme. The high resolution crystal structure models from thermophilic cyanobacteria have fueled recent studies examining the lumenal portion of the PS II complex as a whole for channels that could allow the passage of water, oxygen and/or $\mathrm{H}^{+}$(Bondar and Dau 2012; Vassiliev et al. 2012, 2013; Lorch et al. 2015). Analysis of the PS II crystal structure (Umena et al. 2011) reveals that the interior of the lumenal portion of the PS II complex is quite hydrophilic, containing numerous polar and charged residues and numerous structural waters. These components define extended hydrogen bond networks that potentially allow for the passage of water molecules to, and the transport of $\mathrm{H}^{+}$from, the $\mathrm{Mn}_{4} \mathrm{CaO}_{5}$ cluster (Bondar and Dau 2012).

Recently, a number of studies based on the cyanobacterial PS II crystal structure have implicated PsbO in participating in the formation of an extended hydrogenbonding network(s) at the lumenal surface of the PS II complex. Bondar and Dau (2012) identified a large number of aspartyl/glutamyl clusters, several of which contained structurally resolved water molecules which could participate in such networks. PsbO was suggested to participate in networks involving PsbA, PsbD, PsbC, PsbB, PsbU, and PsbM and it was hypothesized that at least some portion of these networks could serve as proton transport pathways or could couple protonation states with protein conformational changes. The PsbO protein in particular is rich in carboxylate groups, some of which appear to have interesting properties. At least one pair on PsbO, ${ }^{97} \mathrm{E}$ and ${ }^{102} \mathrm{D}$, is close enough to share a proton via a strong hydrogen bond interaction. The carboxylate PsbO: ${ }^{158} \mathrm{D}$ is part of a hydrogen bond network with PsbD residues ${ }^{308} \mathrm{E}$ and ${ }^{310} \mathrm{E}$. Thus, these hydrogen-bonding networks could potentially allow for movement of water molecules and $\mathrm{H}^{+}$and could also contribute to inter-subunit interactions. The PsbU protein also has a number of closely spaced carboxylate residues at its surface that may also contribute to its role in modulating the calcium and chloride requirement in PS II. The PsbV subunit also has residues that have been identified as part of a longer hydrogen bond network connecting the $\mathrm{Mn}_{4} \mathrm{CaO}_{5}$ cluster to the PS II protein surface.

Molecular dynamic simulations of the mobility of water molecules at the surface of PsbO (Lorch et al. 2015) support these suggestions. While there was significant heterogeneity with respect to carboxylate/water bridges on the surface of $\mathrm{PsbO}$, several pairs of residues, ${ }^{84} \mathrm{E} /{ }^{98} \mathrm{E}$, ${ }^{205} \mathrm{D} /{ }^{210} \mathrm{E},{ }^{218} \mathrm{E} /{ }^{232} \mathrm{E}$, and ${ }^{222} \mathrm{D} /{ }^{224} \mathrm{D}$, had high probabilities of carboxylate/water bridging and exhibited slow water dynamics. The ${ }^{222} \mathrm{D} /{ }^{224} \mathrm{D}$ pair continued to show slower water dynamics in further simulations of these pairs, which took into account the differences in relative dynamics between the water molecules and the protein residues and was suggested to be important either structurally, possibly facilitating PsbO binding to PS II, or energetically, as a portion of a proton transfer pathway from the oxygenevolving complex [possibly involving $\mathrm{PsbD}:{ }^{308} \mathrm{D}$ and/or PsbD: ${ }^{310} \mathrm{E}$, see (Fig. 1)]. It was concluded that the high water occupancy at this carboxylate pair may facilitate the 


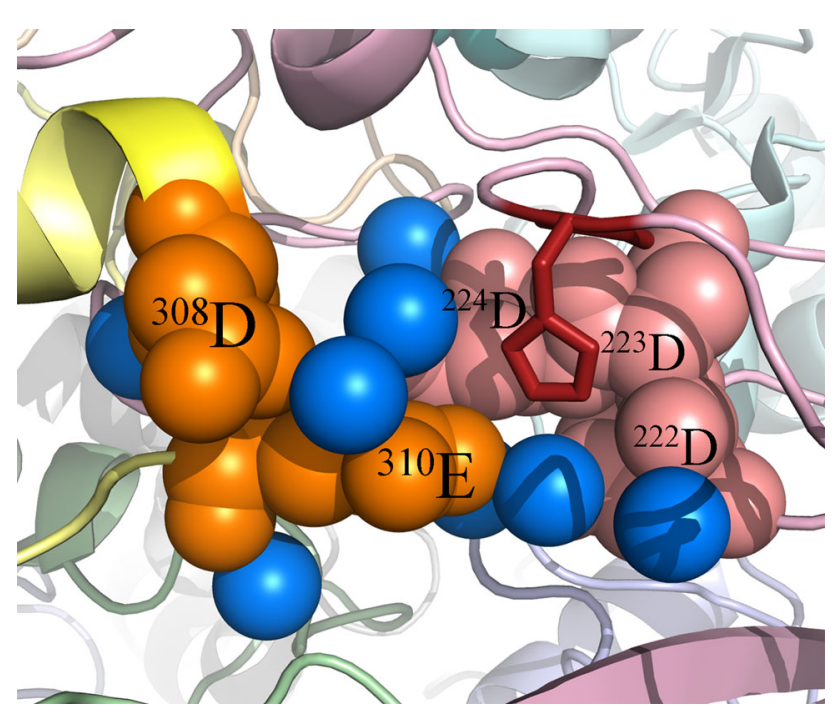

Fig. 1 Organization of a Highly Stable Cluster of PsbO and PsbD Carboxylate Residues in Association with Structural Waters. Identified in Lorch et al. (2015), this cluster may be important either structurally (possibly facilitating PsbO binding to PS II) or energetically (possibly as a portion of a proton transfer pathway from the oxygen-evolving complex to the lumenal surface). PsbO, PsbD, PsbA, PsbC, PsbU and PsbB are shown as ribbons and rendered in pink, yellow, green, blue, cyan, and tan, respectively. The PsbO carboxylate residues ${ }^{222} \mathrm{D},{ }^{223} \mathrm{D}$, and ${ }^{224} \mathrm{D}$ are labeled and shown as salmon spheres. The PsbD carboxylates ${ }^{308} \mathrm{D}$ and ${ }^{310} \mathrm{E}$ are labeled and shown as orange spheres. Waters associated with these carboxylates are shown as blue spheres. PsbO: ${ }^{228} \mathrm{H}$, which is analogous to higher plant PsbO: ${ }^{223} \mathrm{~K}$, is adjacent to this water-carboxylate residue cluster (Pigolev et al. 2012) and is in van der Waals contact with two structural waters of this cluster, is shown as red sticks. All figures were prepared using PyMOL (The PyMOL Molecular Graphics System, Version 1.7.4 Schrödinger, LLC)

docking of the PsbO protein to the PS II complex, as these residues are part of an extended hydrogen-bonding cluster with residues from the PsbA and PsbD proteins. However, it should be noted that simulations including all of these components are not feasible. Earlier, Shutova et al. (Shutova et al. 2007) had suggested that these aspartate/glutamate clusters might act as a buffering network which would efficiently accept protons from the oxygen-evolving site. These hypotheses are not necessarily mutually exclusive. These computational studies provide important guidance, focusing on specific amino acid residues as targets for additional biochemical experiments, particularly site-directed mutagenesis and/or isotope-edited FTIR studies to evaluate their effect on PsbO binding and oxygen-evolving activity. It should be noted that in early biochemical studies, the accessibility of PsbO carboxylates to chemical modification with 1-ethyl-3-(3-dimethylaminopropyl)carbodiimide (EDC) and glycine methyl ester was examined. The ${ }^{222} \mathrm{D}$ and ${ }^{224} \mathrm{D}$ residues were labeled when PsbO was in solution and not when it was bound to PS II (Frankel et al. 1999). These findings are consistent with the interpretation that the ${ }^{222} \mathrm{D} /{ }^{224} \mathrm{D}$ carboxylate pair may be important for the association of PsbO to PS II.

Direct observation of changes in the conformation of PsbO during the S-state cycling has been reported (Offenbacher et al. 2013). Globally labeled ${ }^{13} \mathrm{C}-\mathrm{PsbO}$ was reconstituted with a highly active and purified higher plant PS II core preparation and examined in double-difference FTIR (Fourier Transform Infrared) spectroscopy experiments (light minus dark spectra followed by $S_{n}$ minus $\mathrm{S}_{\mathrm{n}-1}$ ). This study indicated that during the $\mathrm{S}_{1} \Rightarrow \mathrm{S}_{2}$ state transition, conformational alterations were observed upon $\mathrm{PsbO}$ removal. Isotope-edited spectra $\left({ }^{12} \mathrm{C}-\mathrm{PsbO}\right.$ minus ${ }^{13} \mathrm{C}$-PsbO) demonstrated that the structure of $\mathrm{PsbO}$ is altered during the $S_{1} \Rightarrow S_{2}$ state transition. PsbO structural changes on the $S_{2} \Rightarrow S_{3}$ and $S_{3} \Rightarrow S_{0}$ transitions also were observed. It was proposed that the observed changes were due to modulation of the hydrogen-bonding network during S-state transitions. This reported conformational sensitivity of PsbO to S-state cycling indicates tight coupling between the manganese cluster and the extended hydrogen-bonding network and is fully consistent with the proposals described above (Shutova et al. 2007; Bondar and Dau 2012; Lorch et al. 2015).

Molecular dynamics simulations have been used to evaluate the PS II structure for $\mathrm{H}_{2} \mathrm{O}$ and $\mathrm{O}_{2}$ channels from the $\mathrm{Mn}_{4} \mathrm{CaO}_{5}$ cluster to the lumenal surface of PS II (Vassiliev et al. 2012). Residues of the lumenal extrinsic proteins contribute to the entrances of the identified $\mathrm{H}_{2} \mathrm{O}$ channels (Vassiliev et al. 2012). Each of the identified channels also has points of restriction that serve as energetic barriers for the passage of water molecules. In some of these channels, residues of the PsbO, PsbU and PsbV proteins serve as permeation barriers. While the energetic cost is not significant enough to limit water supply to the $\mathrm{Mn}_{4} \mathrm{CaO}_{5}$ cluster, it does provide a molecular explanation for the mechanism by which the lumenal extrinsic proteins protect the $\mathrm{Mn}_{4} \mathrm{CaO}_{5}$ cluster from small reductants. In another simulation study to discover oxygen channels within PS II, significant overlap was discovered with the previously identified putative water channels (Vassiliev et al. 2013). However, there were key differences in the energetics of permeability of water and oxygen. The water permeability restriction points typically did not have the same effect on oxygen. Thus, the same channel could selectively allow the passage of water but also the unrestricted exit of oxygen.

While a large body of biochemical evidence indicates that the lumenal extrinsic subunits of PS II modulate the calcium and chloride requirement for PS II oxygenevolving activity, none of the PS II structural models show a direct connection between any of the extrinsic proteins and the location of these cofactors within the active site (Bricker et al. 2012). Recently, Nagao and coworkers 
(2015) used FTIR difference spectroscopy to examine changes in PS II structure in the vicinity of the $\mathrm{Mn}_{4} \mathrm{CaO}_{5}$ cluster occurring upon removal and reconstitution of the PsbO, PsbU and PsbV subunits. This study dissected the individual structural contributions of the different extrinsic protein subunits and their connection to the calcium and chloride requirement (Nagao et al. 2015). PS II complexes were depleted of the extrinsic subunits (PsbO, PsbU and PsbV) and FTIR difference spectroscopy of the $S_{2}$ minus $\mathrm{S}_{1}$ states was used to monitor changes in the amide I and amide II bands. These bands are sensitive to structural changes in the vicinity of the $\mathrm{Mn}_{4} \mathrm{CaO}_{5}$ cluster other than those associated with the carboxylate and imidazole $\mathrm{CN}$ stretching modes (which are assigned to manganese cluster ligands). These include $\mathrm{CO}$ stretching modes (amide I, $1700-1600 \mathrm{~cm}^{-1}$ ) and $\mathrm{NH}$ bending and $\mathrm{CN}$ stretching modes (amide II, $1600-1500 \mathrm{~cm}^{-1}$ ) of the peptide bond. Consequently, these features monitor the secondary structure organization of the polypeptide backbone in the vicinity of the $\mathrm{Mn}_{4} \mathrm{CaO}_{5}$ cluster.

Removal of the extrinsic proteins (in the presence of $5 \mathrm{mM} \mathrm{CaCl}_{2}$ ) induced the loss of numerous features in the $1700-1300 \mathrm{~cm}^{-1}$ region, with significant changes in both the amide I and II bands. These features were, in large measure, recovered upon reconstitution with the extrinsic subunits. Rebinding of the PsbO protein resulted in the recovery of most of the features which were present in the native conformation, with smaller additional contributions observed upon the addition of the PsbU and PsbV proteins (Nagao et al. 2015). While the PsbV protein could bind independently of the PsbO protein, its association did not correlate with any specific conformational recovery. These results highlight a difference among organisms that contain different extrinsic protein components associated with PS II. In spinach PS II, reconstitution with PsbP was associated with recovery of the native conformation (see below), while in red algae, PsbV binding was found to have the greatest effect (Tomita et al. 2009; Uno et al. 2013). Consequently, photosynthetic organisms can differ significantly in the specific contributions of the individual extrinsic subunits to the protein structure in the vicinity of the $\mathrm{Mn}_{4} \mathrm{CaO}_{5}$ cluster, but the collective effect of the extrinsic proteins appears similar.

In the absence of any extrinsic proteins, increasing the $\mathrm{CaCl}_{2}$ concentration to $100 \mathrm{mM}$ moderated the loss of the amide I and amide II features, which was consistent with some stabilization of the native conformational state by these inorganic cofactors, alone (Nagao et al. 2015). This result indicates that both the extrinsic proteins and high non-physiological $\mathrm{CaCl}_{2}$ concentrations can structurally stabilize PS II and highlights the collective function of the extrinsic proteins to modulate the calcium and chloride requirements for the photosystem. It should be noted that this study assessed only the structural effects of the extrinsic proteins and cofactors in the $S_{1}$ minus $S_{2}$ states of the $\mathrm{Mn}_{4} \mathrm{CaO}_{5}$ cluster and did not examine possible changes occurring in the other $\mathrm{S}$ states. The observation that removal of subsets of the extrinsic proteins in these different systems causes changes in the secondary structure in the vicinity of the $\mathrm{Mn}_{4} \mathrm{CaO}_{5}$ cluster suggests a mechanism for the observed change in the cofactor requirements. Alteration of the secondary structure may affect the accessibility of the calcium and chloride ions to their individual binding sites and/or modify their dissociation constants. Obviously, the identification of the actual location of these secondary structural changes could help differentiate between these and other possibilities.

Recently, an in vivo site-directed mutagenesis system for the PsbO protein has been developed in Chlamydomonas reinhardtii (henceforth Chlamydomonas) [reviewed in (Pigolev and Klimov 2015)]. While site-directed mutagenesis has been used extensively in studying cyanobacterial PsbO in vivo and heterologously expressed eukaryotic PsbO in vitro, no eukaryotic model was available to examine eukaryotic PsbO mutants in vivo. Such a system would be useful to examine the effects of targeted mutations on PS II assembly, particularly with respect to interactions with other PS II components and assembly factors. Chlamydomonas appears to provide a near ideal system for in vivo studies on PsbO, as it can be grown under heterotrophic conditions, is haploid, can be transformed relatively easily and contains only one gene encoding PsbO. When grown heterotrophically and in the dark, $\Delta$ PsbO strains can assemble PS II and these reaction centers can carry out charge separation with the concomitant generation of significant levels of variable fluorescence (Pigolev et al. 2009). Growth in the light apparently leads to rapid photoinactivation and dissasembly of the PS II complex in the absence of PsbO. Using this system (Pigolev et al. 2012), two site-directed mutants have been produced in Chlamydomonas, K223E and K226E. These lysyl residues are homologous to ${ }^{228} \mathrm{H}$ and ${ }^{231} \mathrm{H}$ in Thermosynechococcus vulcanus (henceforth, T. vulcanus). Both of these mutations affect PS II structure and/or function. The K223E mutant accumulated wild-type levels of PsbO, while only small amounts of the protein accumulated in the K226E mutant. It was hypothesized that the modified PsbO in the $\mathrm{K} 226 \mathrm{E}$ mutant may have been improperly folded or could not associate productively with the PS II core, leading to rapid turnover of the photosystem. The K223E mutant could evolve oxygen at about $80 \%$ wild-type rates when grown in the light but exhibited defects in charge recombination as measured by fluorescence decay. In the absence of DCMU, decay was similar to wild type while, in the presence of DCMU, fluorescence decay was significantly slower. This indicated that charge recombination 
between $\mathrm{Q}_{\mathrm{A}}^{-}$and the higher $\mathrm{S}$ states was impaired. The authors speculated that this change might indicate that ${ }^{223} \mathrm{~K}$ was involved in the binding of chloride or $\mathrm{HCO}_{3}{ }^{-}$to a channel leading from the lumen to the $\mathrm{Mn}_{4} \mathrm{Ca} \mathrm{O}_{5}$ cluster. It should be pointed out that in $T$. vulcanus the corresponding residue ${ }^{228} \mathrm{H}$ is in van der Waals contact with the stable cluster of carboxylate residues (PsbO residues ${ }^{222} \mathrm{D}$, ${ }^{223} \mathrm{D}$ and ${ }^{224} \mathrm{D}$, PsbD residues ${ }^{308} \mathrm{D}$ and/or ${ }^{310} \mathrm{D}$ ) and structural waters described above (Lorch et al. 2015), and may participate in stabilizing this highly acidic cluster (Fig. 1). Necessarily, these results must be interpreted cautiously, as charge-swap mutations can lead to large structural and functional perturbations which may or may not be directly associated with the function of the specific targeted residue. Nevertheless, these results are interesting and certainly establish this as a viable system for the sitedirected mutagenesis of PsbO in a eukaryotic system. The interpretation of the phenotypes of such mutants can be problematic, however. Site-directed mutagenesis of a single residue, for instance, might engender a very nuanced phenotype, particularly at the distal residues in a proton conductance or substrate water pathway. Similarly, if multiple residues are involved in PsbO binding, the alteration of a single residue might have little overall effect. The site-directed modification of multiple residues, while possibly leading to more observable phenotypes, also increases the possibility of global structural rearrangement of the protein target. One possible way to circumvent this problem is to examine the fitness of strains bearing a single mutation in competition with a strain carrying the appropriate wild-type allele control. Even very slight changes in fitness will be evident in growth competition experiments of mutant versus wild type. Importantly, the careful biophysical examination of these mutants is imperative.

Arabidopsis contains two forms of PsbO that appear to have different functions (Bricker et al. 2012). This multiplicity of PsbO isoforms (usually two) appears to be a general feature of many angiosperms (Duchoslav and Fischer 2015). Examination of forty-nine species indicated that most of these contained multiple genes encoding PsbO (it should be noted that the expression of these genes has generally not been determined). Surprisingly, $p s b \mathrm{O}$ duplication appears to have occurred independently at or near the roots of most angiosperm families. That these duplications have been maintained suggests that the different PsbO forms may perform different functions. Across all of these lineages, the domain of PsbO which interacts, based on the T. vulcanus crystal structure (Umena et al. 2011), with the intrinsic components of the photosystem is highly conserved. Most of the interfamily and interspecies variability is localized to the $\beta 5-\beta 6$ loop and a portion of the $\beta 6$ strand (T. vulcanus, $\left.{ }^{149} \mathrm{P}-{ }^{197} \mathrm{I}\right)$. Within the various lineages, inter-isoform differences are confined mostly to the $\beta 1-\beta 2$ loop (T. vulcanus, $\left.{ }^{54} \mathrm{~K}-{ }^{69} \mathrm{~T}\right)$ and the end of the $\beta$ barrel extending into the lumen. The observed differences between the isoforms was proposed to be the result of parallel evolution.

The use of mass spectrometry has allowed the identification of post-translational phosphorylation and oxidative modifications on a number of PS II components [for review, see (Bricker et al. 2015)]. In animals, the selective nitration of protein tyrosyl residues is well established (Ischiropoulos 2009) and has been suggested to play roles in signaling pathways involved in redox control mechanisms (Trachootham et al. 2015). Earlier, using a proteomics approach in Arabidopsis, the nitration sites on a number of PS II proteins were identified after Arabidopsis plants were exposed to high light conditions (Galetskiy et al. 2011). These included the extrinsic proteins PsbO-1, PsbO-2, PsbP-1, PsbQ-1 and PsbR as well as a number of intrinsic PS II components. This study did not report quantitative results, however, consequently the extent of modification of these proteins is unclear. Recently, post-translational nitration of tyrosyl residues has been examined in Arabidopsis (Takahashi et al. 2015). Interestingly, when leaves were exposed to $40 \mathrm{ppm} \mathrm{NO}_{2}$ in the light, a strikingly specific pattern of nitration was observed. Analysis of whole leaf protein indicated that virtually all of the protein nitration observed was in the PsbO-1, PsbO-2 and PsbP-1 proteins. When thylakoid proteins were isolated, these three proteins still accounted for the vast majority of observed nitrosylated proteins. While the exogenously supplied $\mathrm{NO}_{2}$ concentrations in this study were very high and non-physiological $(\approx 1000 \times$ ambient levels $)$, the concentration of endogenously produced $\mathrm{NO}_{2}$ in plants is unknown. Interestingly, even in the absence of treatment with $\mathrm{NO}_{2}$, detectable nitrosylation of proteins that comigrated with PsbO-1 and PsbP-1 was observed. While these were not identified rigorously to be PsbO-1 and PsbP-1, this observation may indicate that these PS II components are natively nitrosylated under physiological conditions. Within the context that $\mathrm{NO}_{2}$ may act as a plant growth regulator (Takahashi et al. 2014) at physiological concentrations (10-20 ppb, controlling cell proliferation and enlargement), these results are quite intriguing.

\section{PsbU and PsbV proteins of cyanobacteria}

As noted above, PsbV appears to play an accessory role in stabilizing the native conformation of the PS II core at physiological calcium and chloride concentrations. A recent study of PsbC mutants further examined the connection between the association of PsbV with PS II and the chloride requirement (Burch et al. 2012). Earlier, the PsbC 
mutant ${ }^{1}$ R320S had been described as having defects under low chloride growth conditions due to reduced binding of the PsbV protein (Bricker et al. 2002; Young et al. 2002). PsbC: ${ }^{320} \mathrm{R}$ is located in the large extrinsic loop $\mathrm{E}$ of PsbC and is adjacent to $\mathrm{PsbV}$ residues ${ }^{49} \mathrm{~N}$ and ${ }^{51} \mathrm{~S}$, possibly forming a hydrogen bonding network (PsbV: ${ }^{49-}$ $\left.\mathrm{N} \cdots \mathrm{PsbC}:{ }^{320} \mathrm{R} \cdots \mathrm{PsbV}:{ }^{51} \mathrm{~S}\right)$. Additional mutations at this locus yielded the R320K and R320D mutants which were characterized with respect to PsbV binding and growth under low chloride conditions. The R320D mutant was shown to have severe defects in growth and PS II function under chloride-limiting growth conditions, while the R320K mutant exhibited an intermediate phenotype. Interestingly, PS II complexes isolated from these strains uniformly lost the PsbV protein. Thus, even a conservative substitution in the R320K mutant resulted in a significant destabilization of the PsbV protein's association with the photosystem.

Additional possibilities for the role of PsbV have also been suggested based on a new analysis of the redox potential of this cytochrome (Guerrero et al. 2011; Roncel et al. 2012). Using an alternative titration method which did not rely on the presence of mediators, the midpoint potential of PsbV bound to PS II was much higher than previous estimates $(+200 \mathrm{vs} .-80 \mathrm{mV})$. The possibility of interaction between PsbV and its nearest redox neighbor, the $\mathrm{Mn}_{4} \mathrm{CaO}_{5}$ cluster, has been discussed in recent reviews (Bricker et al. 2012; Roncel et al. 2012). Biochemical experiments to probe this possibility are still lacking. Given the amount of structural data and the biochemical tools available for studying this protein in cyanobacteria, it is likely that studies of site-directed PsbV mutations which alter its redox potential without altering binding may be forthcoming. It is also noteworthy that the measured midpoint potential of the free protein $(-240 \mathrm{mV})$ is significantly different from that of the PS II-bound PsbV $(+200 \mathrm{mV})$ (Roncel et al. 2012). These data strongly suggest that the physical association of PsbV with the photosystem dramatically affects its midpoint potential. It would be very interesting to determine what, if any, changes in the midpoint potential of PsbV occur in PS II complexes lacking PsbO, PsbU, or combinations of these proteins. Furthermore, studies examining the effects on midpoint potential under different calcium and chloride conditions, in the presence or absence of other extrinsic protein components, seem quite feasible.

A new study on the $\triangle p s b U$ mutant in Synechococcus sp. PCC 7942 (henceforth, Synechococcus) has highlighted

\footnotetext{
${ }^{1}$ Using the Synechocystis PCC sp. 6803 numbering system, these mutants were originally designated R305K and R305D. In this article, unless otherwise specified, we will follow the common practice of numbering the amino acid residues using the T. vulcanus numbering system.
}

specific contributions of the PsbU protein within PS II as well as differences among cyanobacterial species (Abasova et al. 2011). $\Delta p s b U$ mutants have previously been characterized from Synechocystis sp. PCC 6803 (henceforth Synechocystis) and Synechococcus with noted defects in electron transfer through PS II (Shen et al. 1997; Nishiyama et al. 1999; Veerman et al. 2005; Inoue-Kashino et al. 2005; Balint et al. 2006). The most recent study in Synechococcus shows slower charge recombination from both $\mathrm{Q}_{A}$ and $\mathrm{Q}_{B}$ as well as increased light sensitivity, irrespective of the presence or absence of the protein synthesis inhibitor lincomycin (Abasova et al. 2011). Because the effects of the PsbU protein on PS II function appear subtle, it has been difficult to assign a specific role for this protein. Moreover, the careful re-analysis of the Synechococcus $\Delta p s b U$ mutant shows some differences relative to the Synechocystis $\Delta p s b U$ mutant, which underscores possible differences in PS II even between two mesophilic cyanobacteria.

Earlier, a very unusual phenotype was observed for the double deletion mutants $\triangle p s b O \Delta p s b U$ and $\triangle p s b Q \Delta p s b V$. These mutants were unable to grow photoautotrophically at $\mathrm{pH} 7.5$, but growth could be restored at $\mathrm{pH} 10$ (Eaton-Rye et al. 2003; Summerfield et al. 2005). The restoration of photoautotrophy at alkaline $\mathrm{pH}$ has been puzzling because studies at the level of membranes and isolated PS II particles in both spinach and cyanobacteria have established an inhibitory effect of alkaline pH on PS II activity (Kuwabara and Murata 1982; Cole et al. 1986; Schlodder and Meyer 1987). In fact, alkaline lumenal $\mathrm{pH}$ has been shown to result in the release of the extrinsic proteins (Kuwabara and Murata 1982).

Recent characterization of these mutants has revealed a partial explanation for these phenotypes (Summerfield et al. 2013). Changes in PS II function were minor in the two different $\mathrm{pH}$ conditions with no differences in variable fluorescence parameters. There was a measureable difference, however, in phycobilisome antenna coupling to PS II, with decreased coupling being observed at the restrictive $\mathrm{pH}$ (7.5) and increased coupling observed at the permissive $\mathrm{pH}$ (10). The improvement in antenna coupling, however, was not sufficient to fully explain recovery of photoautotrophic growth at $\mathrm{pH} 10$. Interestingly, these mutants were very light-sensitive at $\mathrm{pH} 7.5$, which is consistent with an overall increase in oxidative stress at this $\mathrm{pH}$. Transcriptional analysis revealed that upon shift from the permissive $\mathrm{pH} 10$ to the restrictive $\mathrm{pH} 7.5$, stress-responsive genes were globally downregulated. Consequently, at higher $\mathrm{pH}$, cells may be poised to be less sensitive to oxidative stress and, consequently, they could cope more easily with the increased oxidative stress in the PS II mutants. At $\mathrm{pH} 7.5$, the oxidative stress generated by the PS II defects in the mutants could overwhelm the stress response systems, making photoautotrophic growth 
impossible. This may explain the pH-dependent growth phenotype observed in these strains.

Consistent with this interpretation, wild-type cells were more resistant at $\mathrm{pH} 10$ to treatment with the dye rose bengal, which under illumination generates ${ }^{1} \mathrm{O}_{2}$, than at $\mathrm{pH}$ 7.5 , although a similar trend was not observed for treatment with methyl viologen, which generates $\mathrm{O}_{2}{ }^{-}$. In either case, the $\triangle p s b O \Delta p s b U$ and $\triangle p s b Q \triangle p s b V$ mutants were more sensitive to both rose bengal and methyl viologen (Summerfield et al. 2013). These results underscore the complex pleiotropic effects that mutations in the extrinsic PS II subunits can exhibit. While the pH-dependence phenotype is clearly PS II-dependent, there may be multiple factors at play beyond PS II, and future studies on the oxidative stress responses at different $\mathrm{pH}$ conditions are clearly warranted. Beyond the connection with oxidative stress, numerous physiological changes occur at the level of whole cells upon the $\mathrm{pH}$ shift from 7.5 to 10 . It is possible there are changes in other factors, such as transporters, which alter the intracellular ionic environment to be more favorable for photoautotrophy in these mutants.

\section{The CyanoQ and CyanoP proteins of cyanobacteria}

Strong evidence has been presented for the association of the CyanoQ with PS II in the mesophilic cyanobacterium Synechocystis, but it remains absent from all of the crystal structures currently available from the thermophilic cyanobacteria (Thornton et al. 2004; Roose et al. 2007b; Umena et al. 2011). Consequently, its position within the photosystem cannot be directly observed and its general significance in cyanobacterial PS II function is under debate. Since the last comprehensive review of the PS II extrinsic proteins, significant progress has been made toward resolving these apparent disparities between mesophilic and thermophilic strains.

Recently, CyanoQ was detected in thylakoids and PS II complexes from the thermophilic cyanobacterium Thermosynechococcus elongatus (henceforth, T. elongatus) (Michoux et al. 2014). The CyanoQ protein was enriched in PS II complexes, relative to thylakoid membranes, to a similar degree as that of the PsbD protein, but semiquantitative analysis revealed a ratio of 0.4 CyanoQ per PS II monomer. This differs from estimates of CyanoQ in Synechocystis, which range from 1.0 CyanoQ per PS II monomer, based on thylakoid membranes signals, to 0.3 CyanoQ per PS II monomer in CyanoQ-histidine-tagged complexes. This variation is likely the result of differences in sample treatments since the T. elongatus PS II particles were purified using a histidine-tagged $\mathrm{PsbC}$ protein followed by a two-step anion-exchange purification procedure. Additional treatment of the T. elongatus PS II complexes similar to the conditions used for PS II crystallization resulted in the loss of CyanoQ from the complexes, which is consistent with its absence from the current PS II crystal structures.

The structure of T. elongatus CyanoQ also was reported [PDB:3ZSU, (Michoux et al. 2014)]. Overall, it is similar to the four-helix-bundle structure of Synechocystis CyanoQ [PDB:3LS0 and 3LS1, (Jackson et al. 2010)] as well as PsbQ from higher plants (PDB: 1NZE (Calderone et al. 2003) and PDB:1VYK (Balsera et al. 2005). While the Synechocystis CyanoQ structure contained two zinc atoms, a zinc-containing CyanoQ structure from $T$. elongatus could not be obtained despite the fact that the majority of the zinc ligand residues are conserved between the two species. The functional significance, if any, of these cation binding sites within CyanoQ remains unknown.

While CyanoQ is absent from cyanobacterial PS II crystal structures, recently Liu et al. (2014) have used chemical crosslinking and tandem mass spectrometry data from Synechocystis to map the location of CyanoQ within the photosystem (Liu et al. 2014). The CyanoQ protein was found to be crosslinked to both the PsbO and PsbB subunits, consistent with a position at the PS II dimer interface. Additionally, crosslinked products between CyanoQ peptides were interpreted as resulting from intermolecular crosslinking between two CyanoQ subunits, suggesting a close antiparallel arrangement of CyanoQ subunits at the PS II dimer interface (Fig. 2). This structural arrangement is consistent with biochemical evidence demonstrating that CyanoQ is associated with dimeric PS II complexes containing the full complement of extrinsic proteins, but absent from PS II complexes lacking the PsbO protein (Liu et al. 2014). It will be interesting to compare this predicted position of CyanoQ with any forthcoming CyanoQ-containing thermophilic PS II crystal structures to determine if there are significant conformational changes between the free and PS II-bound forms of CyanoQ. It should be noted that in Synechocystis and T. elongatus, CyanoQ contains a lipid modification at its $\mathrm{N}$-terminus that tethers the otherwise soluble protein to the thylakoid membrane (Fagerlund and Eaton-Rye 2011). It is unclear from the currently predicted CyanoQ position at the PS II dimer interface if the N-terminus can extend to the lipid bilayer (Fig. 2).

The study described above has been instrumental in identifying the location of the PsbQ subunit within mature cyanobacterial PS II. Recently, additional work by Liu and coworkers (2015) has added another level of complexity with respect to the association of CyanoQ with the photosystem (Liu et al. 2015). Previous studies have indicated that there was a single CyanoQ associated per PS II monomer (Thornton et al. 2004; Roose et al. 2007b; Liu et al. 2014). Further characterization of histidine-tagged CyanoQ PS II has identified an additional CyanoQ-containing PS II complex which contains multiple copies of 


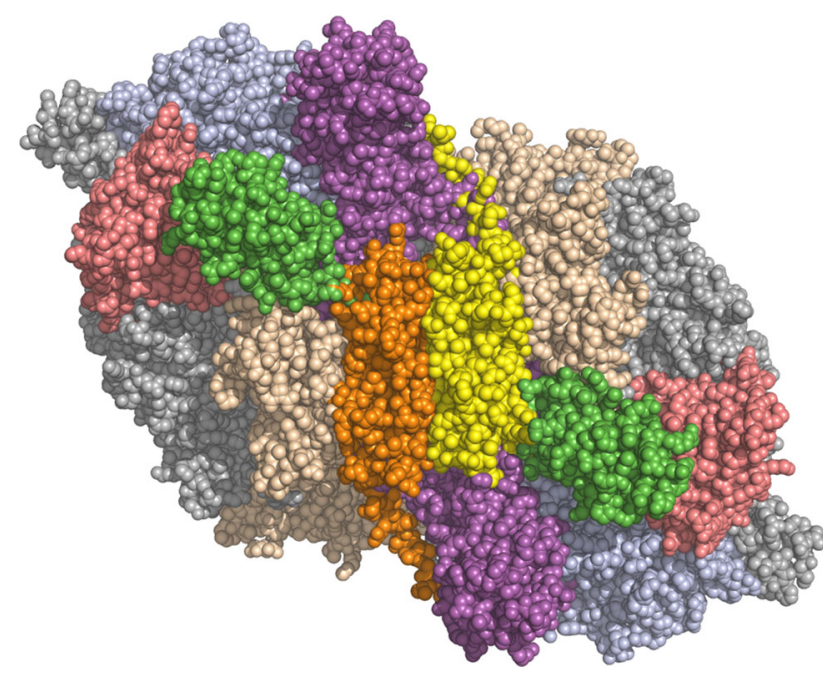

Fig. 2 Proposed Location of CyanoQ within the Cyanobacterial PS II Complex based on Protein Crosslinking and Tandem Mass Spectrometry. This is redrawn from Liu et al. (2014). This view is from the lumen looking down onto the PS II dimer. The two CyanoQ subunits (shown in orange and yellow) are each associated with one PS II monomer and are positioned at the monomer-monomer interface within the dimeric PS II structure. The extrinsic subunits PsbO, PsbU and PsbV are shown in purple, green and pink, respectively. The intrinsic subunits $\mathrm{PsbB}$ and $\mathrm{PsbC}$ are shown in tan and pale blue, respectively. The $\mathrm{N}$-terminal domains of $\mathrm{CyanoQ}$, are shown as highly extended strands adjacent to the PsbO subunits

CyanoQ per PS II monomer (Liu et al. 2015). This new complex elutes from nickel-affinity columns at higher imidazole concentrations than the previously characterized CyanoQ-containing PS II complex. These dimeric PS II complexes have a unique extrinsic protein composition, in that they lack the PsbU and PsbV proteins, but contain an estimated four copies of CyanoQ. These complexes have reduced oxygen-evolving activity and this observation complicates previous interpretations of CyanoQ function in PS II. Previous results were consistent with a role for CyanoQ as a stoichiometric subunit that is incorporated into the complex late during assembly (Roose et al. 2007b). However, this new complex suggests a role for CyanoQ in the assembly and/or turnover of the photosystem. Liu et al. (2015) presented a model in which multiple copies of CyanoQ associate with PS II during assembly, but during PS II complex maturation a subset of these copies dissociate. The dynamic nature of PS II assembly and repair is complex; nevertheless, it is difficult to imagine an underlying molecular mechanism that could temporally exchange such a profoundly different complement of extrinsic protein subunits.

The CyanoP protein is a homolog of the well-characterized PsbP protein in higher plants. CyanoP has been identified in numerous cyanobacterial species, but it remains the most enigmatic extrinsic subunit with respect to its role in cyanobacterial PS II (Bricker et al. 2012,
2013). Several different AcyanoP mutants have been characterized from different laboratories and differences among these mutants is evident (Thornton et al. 2004; Ishikawa et al. 2005; Summerfield et al. 2005; Sveshnikov et al. 2007; Abasova et al. 2011). Phenotypically, the effect of the loss of CyanoP is very subtle even when probed under exacerbating conditions like limited $\mathrm{CaCl}_{2}$.

A recent study characterizes a complete deletion mutant in Synechocystis and provides additional information on the $\Delta$ cyanoP mutant phenotype (Aoi et al. 2014). Measurable but subtle effects in photoautotrophic growth and PS II function were detected under $\mathrm{CaCl}_{2}$-limiting conditions. Additionally, there was also a notable change in wild-type cells following subculturing under $\mathrm{CaCl}_{2}$-limiting conditions, ultimately yielding little difference between the wild-type cells and $\triangle$ cyanoP mutant cells under these cofactor-limiting conditions. Apparently, the presence of CyanoP in the wild-type cells was able to delay the onset of defects associated with $\mathrm{CaCl}_{2}$ limitation; ultimately, however, both wild type and $\triangle$ cyanoP show equivalent oxygenevolving deficiencies. While there was no overall decrease in PS II content, measurable defects in coupling of the phycobilisome to the reaction centers were observed in $77 \mathrm{~K}$ fluorescence measurements. Since CyanoP is a lumenal subunit, it is physically distant from the phycobilisomes, which reside on the cytoplasmic side of the thylakoid membrane. Consequently, this effect on energetic coupling is a long distance, transmembrane phenomenon. Similar energy transfer defects have been reported for a $\triangle p s b U$ mutant, suggesting the propagation of conformational changes over long distances within the complex (Veerman et al. 2005). These transmembrane effects are similar to those observed in higher plants upon removal of PsbP and PsbQ, which leads to defects in reducing-side electron transport (Roose et al. 2010).

The detection of CyanoP in thykaloid membranes and PS II complexes has been technically challenging, and conflicting results have been reported (Thornton et al. 2004; Ishikawa et al. 2005). In an attempt to identify conditions that stabilize the association of CyanoP with the PS II complex, Aoi et al. (2014) have examined different detergents for thylakoid solubilization. The detergent $n$ dodecyl- $\beta$-D-maltoside is normally used for solubilizing cyanobacterial membranes for PS II purification; however, this detergent performed poorly, with most of the CyanoP protein being lost from PS II. Both $n$-dodecyl- $\alpha$-D-maltoside and $n$-heptyl- $\beta$-D-thioglucoside detergents more readily solubilized the CyanoP protein as part of a larger protein complex (Aoi et al. 2014). Nevertheless, after solubilization of thylakoid membranes with $0.7 \% n$-dodecyl- $\alpha$-D-maltoside, only a minor fraction of CyanoP appeared associated with a PS II complex which contained at least the PsbD protein (the only PS II protein that was 
tested) after gel filtration analysis; the majority of CyanoP was detected as free protein. Perhaps the use of alternative solubilization protocols will facilitate CyanoP detection and purification of CyanoP-associated PS II complexes.

In general the small but measureable effects on PS II in the absence of CyanoP are consistent with the loss of a stoichiometric subunit (Bricker et al. 2013). In contrast, the difficulty detecting CyanoP within the PS II complex and the initial report of its low abundance $(\sim 3 \%$ in thylakoids relative to PsbB) are more consistent with a possible role for CyanoP in PS II assembly (Thornton et al. 2004; Aoi et al. 2014). Combination mutants can often give additional evidence for a protein's function by exacerbating the observable phenotype. Jackson and Eaton-Rye (2015) have taken this approach with respect to CyanoP function in PS II assembly in Synechocystis by characterizing the $\Delta c y a$ noP $\Delta y c f 48$ mutant relative to the individual mutants $\Delta c y a n o P$ and $\Delta y c f 48$ (Jackson and Eaton-Rye 2015). Ycf48 is involved in PS II assembly and is associated with PS II pre-complexes (Komenda et al. 2008; Jackson et al. 2014). In the analysis of the $\Delta$ cyanoP $\Delta y c f 48$ mutant, the phenotype was dominated by defects observed in $\Delta y c f 48$, but there was evidence that there was an additional alteration in the energetic coupling of the phycobilisome antenna upon loss of CyanoP (Jackson and Eaton-Rye 2015). Analysis of the PS II complexes accumulating in the double mutant in comparison to the single mutants and the control strain revealed an increase in the amount of assembled PS II in the $\Delta$ cyanoP $\Delta y c f 48$ mutant relative to the $\Delta y c f 48$ mutant, indicating that the presence of CyanoP may hinder PS II assembly in the absence of Ycf48. However, these effects are very small and conditions have yet to be found where CyanoP plays a significant role in PS II assembly.

Elucidation of the CyanoP structure has also been the subject of recent studies. The solution NMR structure of CyanoP from Synechocystis has been recently reported [PDB:2LNJ, (Jackson et al. 2012)]. This structure structurally resolves loop regions and the N-terminus, which were not identified in the crystal structure of CyanoP from T. elongatus [PDB:2XB3, (Michoux et al. 2010)]. However, an N-terminal domain corresponding to residues 24-31 still remained highly disordered in solution and no consensus structural model for this domain could be predicted. A number of zinc or manganese atoms have been observed in the crystal structures of CyanoP (these cannot be observed in NMR) as well as PsbP from higher plants (Ifuku et al. 2004; Michoux et al. 2010; Kopecky et al. 2012; Cao et al. 2015). There is no evidence for these potential divalent cation binding sites beyond the crystal structure models, but the sites are differentially conserved among PsbP family members. Based on the presence of conserved ligand residues, at least two of these sites may be of physiological significance in cyanobacteria (Jackson et al. 2012). While these have been correlated with hypotheses regarding PS II functional deficiencies under $\mathrm{CaCl}_{2}$-limiting conditions in the absence of CyanoP, as well as manganese-binding by PsbP in higher plants (Bondarava and Krieger-Liszkay 2007; Cao et al. 2015), no experimental evidence has been presented to show the functional significance of divalent cation-binding properties by CyanoP.

Directly mapping the binding site of CyanoP onto cyanobacterial PS II complexes has been impossible due to issues with low stoichiometry and loss of the protein during PS II isolation. Cormann and colleagues (2014) addressed this problem using a combination of in vitro techniques (Cormann et al. 2014). Surface plasmon resonance (SPR) was used to determine the interaction of CyanoP and other lumenal extrinsic proteins, with protein portions corresponding to lumenally exposed loop regions of PsbA, PsbD, PsbC, PsbB and the PsbO protein. In this artificial system, appropriate positive control associations between PsbV with PsbC and PsbO with PsbC, PsbA and PsbD were observed; negative controls, the lack of association between proteins, were generally in agreement with the crystal structural models. It must be noted that the observed dissociation constants for the PsbO association was considerably higher $(\mu \mathrm{M})$ than previously published values for PsbO (nM) (Leuschner and Bricker 1996), in which PsbO was reconstituted to the full PS II complex and not loop domains of individual proteins. In the SPR experimental system, the CyanoP protein was found to associate with the $\mathrm{C}$-terminal domain of the PsbD protein and the PsbA a-loop, which corresponds to a position roughly analogous to that of PsbO within the PS II structure. Additional reconstitution experiments with CyanoP showed that the protein could bind to monomer PS II complexes lacking the PsbO, PsbU and PsbV proteins but not to dimeric PS II containing the full complement of extrinsic proteins. Based on these data, the CyanoP protein would appear to be involved in PS II assembly rather than as a stoichiometric subunit within the complex. Given the complex nature of the structural relationships among the extrinsic proteins of PS II, additional experiments on CyanoP binding are clearly necessary.

Many outstanding questions remain regarding the CyanoP protein in PS II. The issue of CyanoP abundance within the thylakoid membranes and PS II remains unsettled. Clearly, CyanoP is not enriched in PS II complexes relative to membranes samples, but this may be an artifact of the detergents required to solubilize PS II complexes from the membranes and the susceptibility of the N-terminally lipid-modified CyanoP protein to dissociate from the complex under these isolation conditions (Aoi et al. 2014). The issue of CyanoP abundance within the 
membranes has not been revisited since the two initial conflicting reports were published (Thornton et al. 2004; Ishikawa et al. 2005). Sources for the apparent discrepancy could be the use of different antibodies with different titers for the CyanoP protein identification as well as the technical differences in the methods used for semi-quantification. Given that the abundance of CyanoP in cyanobacterial membranes could influence the interpretation of data regarding its function in PS II, further quantitative analysis of CyanoP in membranes is warranted.

Extensive analyses of several independently constructed mutants by different research groups have yielded a consensus that the loss of CyanoP produces subtle effects on PS II function (Bricker et al. 2013). As noted above, particularly under conditions of limiting $\mathrm{CaCl}_{2}$, PS II complexes lacking CyanoP appear to have defects consistent with the loss of a stoichiometric subunit. It has also been shown that CyanoP does not accumulate in PS II-less cyanobacterial strains, indicating CyanoP stability in the absence of PS II is compromised (Ishikawa et al. 2005). Alternatively, the limited data on CyanoP stoichiometry and limited observable association with mature PS II are more consistent with CyanoP acting as an assembly factor. Additional characterization of the $\triangle$ cyanoP mutants to specifically probe aspects of PS II assembly is needed. Assays of photoactivation to monitor assembly of the $\mathrm{Mn}_{4} \mathrm{CaO}_{5}$ cluster and PS II repair after photodamage are commonly employed to evaluate the capacity for PS II assembly, yet these assays have not been reported for any of the $\triangle$ cyanoP mutants. Evaluating the $\triangle$ cyanoP mutants for these processes under both nutrient-replete and $\mathrm{CaCl}_{2}-$ limiting conditions should allow for a better understanding of the role of CyanoP, if any, in PS II assembly.

\section{The PsbP and PsbQ proteins of green algae and higher plants}

PsbP appears to be critically important for the maintenance of calcium and chloride at the active site for water oxidation in vitro and it is generally assumed that it plays a similar role in vivo (Bricker and Frankel 2011; Bricker et al. 2012, 2013). The role of PsbQ is more unclear, although its functional and structural interaction with PsbP is the subject of a number of recent studies. As is the case with PsbO, the mechanism by which these proteins support water oxidation is poorly understood. These proteins will be discussed together since there is a growing body of evidence that they interact both structurally and functionally within the PS II complex.

With respect to PsbP, earlier studies indicated that PsbP is required for photoautotrophy (Ifuku et al. 2005; Yi et al. 2007; Ido et al. 2009) and normal thylakoid development (Yi et al. 2009). One aspect of this requirement was puzzling, however. Relatively normal growth rates and assembly of PS II were observed even at sub-stoichiometric amounts of PsbP (Ifuku et al. 2005; Yi et al. 2007; Ido et al. 2009). Complete loss of photoautotrophy was observed only when the amount of PsbP was $<5-10 \%$ of wild-type amounts. This seemed unusual given the generally accepted model, based largely on in vitro studies, that the sole function of PsbP was to directly support the cofactor requirement for oxygen evolution. Recently, in Arabidopsis Col-0, it was found that while T-DNA psbpl plants could not grow photoautotrophically, T-DNA psbq1psbq2 psbr triple mutant plants, which completely lacked detectable PsbP after 4 weeks of growth, grew normally (Allahverdiyeva et al. 2013). These plants did contain small amounts of PsbP at earlier growth stages. Surprisingly, the steady-state level of PsbP was also observed to progressively decrease to low levels in older wild-type Arabidopsis plants. The authors suggested that these results may indicate that PsbP is required in seedlings for initial PS II assembly but may be dispensable in older plants. This possibility had not been examined critically in earlier studies but clearly requires further investigation.

FTIR studies have been used to demonstrate that the binding of PsbP to PS II induced protein conformational changes in the vicinity of the $\mathrm{Mn}_{4} \mathrm{CaO}_{5}$ cluster (Tomita et al. 2009). The removal of PsbP led to alterations in the amide $I$ band of the $S_{2}$ minus $S_{1}$ difference spectra which were reversed upon reconstitution with recombinant PsbP. Similar changes were subsequently observed for the $S_{2} Q_{A}^{-}$ minus $S_{1} Q_{A}$ difference spectra (Ido et al. 2012). No changes were observed in the carboxylate and imidazole $\mathrm{CN}$ stretching bands which had been assigned to manganese cluster ligands. Reconstitution with recombinant ${ }^{13} \mathrm{C}$-PsbP demonstrated that the observed amide I band perturbations arose from changes in the intrinsic core PS II proteins and not changes within PsbP. Highlighting the importance of the N-terminus of PsbP [reviewed in (Bricker et al. 2012)], reconstitution with the N-terminally truncated $\Delta 15$-PsbP protein did not restore the native PS II conformation.

Earlier, chemical modification studies were utilized to examine the binding determinants for PsbP to PS II (Tohri et al. 2004). These authors concluded that basic residues which lie in both the $\mathrm{N}$ - and C-terminal domains of the protein were involved in the association of PsbP with the photosystem. Recently, three basic residues $\left({ }^{48} \mathrm{R},{ }^{143} \mathrm{~K}\right.$ and ${ }^{160} \mathrm{~K}$ ) have been examined by site-directed mutagenesis (Nishimura et al. 2014). Three mutated recombinant proteins were produced, R48A, K143A and K160A. Reconstitution experiments demonstrated that all of the mutated proteins bound poorly to NaCl-washed PS II membranes, were significantly less capable of supporting steady-state oxygen evolution and required higher chloride 
concentrations to support maximal oxygen evolution rates. Additionally, changes in the $\mathrm{S}_{2} \mathrm{Q}_{\mathrm{A}}^{-}$minus $\mathrm{S}_{1} \mathrm{Q}_{\mathrm{A}}$ difference spectra brought about by removal of PsbP were not reversed upon reconstitution with any of the mutated proteins. Finally, all of the mutants exhibited loss of the crosslinking interaction between PsbP: ${ }^{1} \mathrm{~A}$ and $\mathrm{PsbE}:{ }^{57} \mathrm{E}$ (Ido et al. 2012). These results indicate that all three of these mutations altered the ability of PsbP to interact with the PS II core complex.

Major advances have recently been made in our understanding of the structure of PsbP and its integration into PS II. Importantly, a new crystal structure [PDB: 4RTI, (Cao et al. 2015)] is available which clarifies the structure of two previously unresolved domains $\left({ }^{90} \mathrm{~K}-{ }^{107} \mathrm{~V}\right.$ and $\left.{ }^{135} \mathrm{~T}-{ }^{139} \mathrm{D}\right)$ and several additional residues at the N-terminus $\left({ }^{11} \mathrm{P}-{ }^{15} \mathrm{~N}\right)$ in PsbP (Fig. 3). Crystallization of spinach PsbP which had been treated with $\mathrm{MnCl}_{2}$ apparently stabilizes the three-dimensional structure of these domains. Two manganese are present; Mn1 is coordinated to ${ }^{144} \mathrm{H},{ }^{165} \mathrm{D}$ and one chloride while $\mathrm{Mn} 2$ is associated with ${ }^{98} \mathrm{D}$ and a structurally resolved water. The authors proposed that Mn1 is strongly bound and Mn2 is weakly associated with the protein. Both are exposed at the surface of the protein. It should be noted that earlier it had been hypothesized that PsbP could serve as a manganese-carrier protein and had presented evidence that photoassembly of the manganese cluster was accelerated in the presence of PsbP and $\mathrm{MnCl}_{2}$ [(Bondarava et al. 2005; Bondarava and Krieger-Liszkay 2007); for a discussion of these papers see

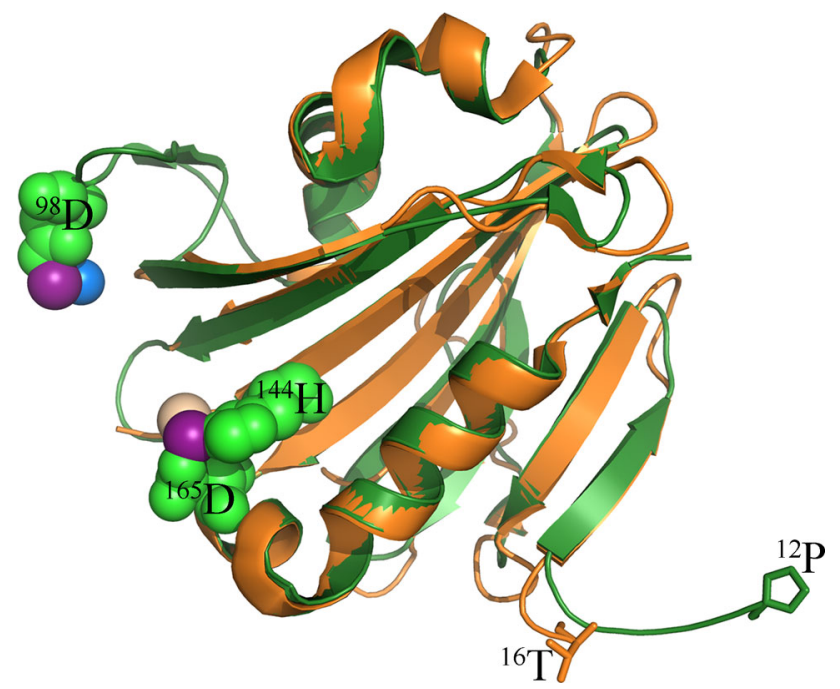

Fig. 3 Comparison of the 2VU4 and 4RTI Crystal Structures of Isolated PsbP. The 2VU4 structure (Kopecky et al. 2012) is shown in orange and the 4RTI (Cao et al. 2015) structure is shown in green. The residues in the 4RTI structure which coordinate Mn1 $\left({ }^{144} \mathrm{H}\right.$ and $\left.{ }^{165} \mathrm{D}\right)$ and $\mathrm{Mn} 2\left({ }^{98} \mathrm{D}\right)$ are shown as bright green spheres. The $\mathrm{N}$-terminal residues, ${ }^{16} \mathrm{~T}$ for $2 \mathrm{VU} 4$ and ${ }^{12} \mathrm{P}$ for $4 \mathrm{RTI}$, are shown as sticks. Manganese are shown as purple spheres, chloride as a tan sphere, and water as a blue sphere
(Bricker and Frankel 2011)]. In the earlier crystal structure [PDB: 2VU4, (Kopecky et al. 2012)] $\mathrm{Zn}^{+2}$ was also found to be ligated to ${ }^{144} \mathrm{H},{ }^{165} \mathrm{D}$ and a structurally resolved water; however, the residues in the unstructured domains were unresolved. At this point in time, it is unclear if the presence of $\mathrm{Mn}^{+2}$ (and or $\mathrm{Cl}^{-}$) at this site is biologically relevant or simply reflects more favorable crystallization conditions. It should be noted that other differences in the crystallization conditions in these studies were present which could, at least theoretically, account for the different structures (for example, for 4RT1, 0.1 M magnesium formate and $15 \%$ w/v PEG3350 was used, while for 2VU4 $16 \%$ PEG monomethylether 550, 0.1 M Tris-HCl, pH 7.5, $10 \mathrm{mM} \mathrm{ZnSO}_{4}$ was used). It should be emphasized that these crystal structures for PsbP are for protein that is not bound to PS II. It is quite possible that substantial conformational changes could occur upon binding of this component to the photosystem.

The role of PsbP: ${ }^{144} \mathrm{H}$ and PsbP: ${ }^{165} \mathrm{D}$ has recently been investigated by site-directed mutagenesis (Ido et al. 2012). Two mutant proteins H144A-PsbP and D165V-PsbP were recombinantly produced and both bound to $\mathrm{NaCl}$-washed PS II membranes with kinetics similar to that observed for control PsbP. The H144A-PsbP reconstituted PS II, however, required $\approx 10 \times$ more chloride for optimal oxygen evolution than did membranes reconstituted with control PsbP protein. Membranes reconstituted with D165V-PsbP did not exhibit a defect in chloride sequestration. Interestingly, the double mutant H144A/D165V exhibited normal chloride binding in support of oxygen evolution. Additionally, FTIR difference spectroscopy indicated that while reconstitution of $\mathrm{NaCl}$-washed membranes with H144A-PsbP did not restore changes in the amide I band brought about by PsbP removal, reconstitution with the H144A/D165V double mutant did restore the amide I band. Finally, protein crosslinking experiments indicated that the interaction of control PsbP: ${ }^{1} \mathrm{~A}$ with PsbE: $:{ }^{57} \mathrm{E}$ was disrupted in the H144A mutated protein but restored in the H144A/ D165V mutated protein. Since the N-terminus of PsbP is critically important for chloride sequestration (Ifuku and Sato 2001), restoration of the core PS II amide I band (Tomita et al. 2009) and interaction of PsbP with PsbE (Ido et al. 2012), the authors concluded that PsbP: ${ }^{144} \mathrm{H}$, which is in the C-terminal domain, is critical for maintaining proper $\mathrm{N}$-terminal interactions with the photosystem.

A critical problem with all of the crystal structures available for PsbP is that the $\mathrm{N}$-terminus of the protein $\left({ }^{1} \mathrm{~A}-{ }^{10} \mathrm{~K}\right)$ is not resolved (Fig. 3). As noted above, this region is particularly important for both the binding of PsbP to the photosystem and for its function in support of oxygen evolution. It has been proposed that PsbP has an extended N-terminus (Ido et al. 2014; Cao et al. 2015) when bound to the PS II complex. In our laboratory, we 
have examined the structure of the PsbP protein when it is bound to the photosystem, using protein crosslinking with bis(sulfosuccinimidyl)suberate (BS3), tandem mass spectrometry, and distance-constrained molecular dynamic refinement of the structure of the N-terminal domain (Mummadisetti et al. 2014). Our results, based on nine independently identified crosslinked products, indicate that the N-terminus of bound PsbP is not extended and that, overall, PsbP assumes a compact structure (Fig. 4). These interactions could not be identified when unbound PsbP was exposed to BS3 in solution. Using crosslinked distance constraints, molecular dynamic structural refinement for the $\mathrm{N}$-terminus indicates that the $\mathrm{N}$-terminus is located 9-18 $\AA$ from ${ }^{144} \mathrm{H}$. These results place strong constraints on the location of PsbP within higher plant PS II (Mummadisetti et al. 2014; Bricker et al. 2015). These results provide experimental verification that the $\mathrm{N}$-terminal domain of PsbP interacts with the C-terminal domain as had been previously proposed (Ido et al. 2012).

The function of PsbQ is quite unclear. In vitro, removal of PsbQ has minor consequences on oxygen evolution except at very low chloride concentrations (Miyao and Murata 1985) and in vivo its absence has no effect on photoautotrophic growth (Ifuku et al. 2005; Yi et al. 2009; Allahverdiyeva et al. 2013) except under low light growth conditions (Yi et al. 2006). As noted above, N-terminal truncation of PsbP results in the dramatic loss of binding to PS II and loss of oxygen-evolving activity at low calcium and chloride concentrations (Ifuku and Sato 2001). Both of these defects can be significantly corrected in the presence of PsbQ (Ifuku and Sato 2002, 2001). Recently, the effects of PsbQ on the interaction of PsbP with PS II have been more carefully examined (Kakiuchi et al. 2012). Reconstitution of either $\Delta 15$-PsbP or the H144A PsbPs onto $\mathrm{NaCl}$-washed PS II membranes in the presence of PsbQ corrected the defects observed in these mutants. In the case of $\Delta 15$-PsbP, binding was fully restored and in both mutants significant increases in oxygen evolution activity were observed. The authors concluded that PsbQ stabilized the interaction of the mutated PsbP proteins with PS II, and that this interaction restored the ability of these proteins to retain both calcium and chloride. Additionally, while neither mutated PsbP protein alone could restore the normal amide I features associated with the oxygen-evolving complex in NaCl-washed membranes, reconstitution of the mutated PsbP proteins in the presence of PsbQ did, in large measure, restore these features. These findings strongly suggest that PsbP and PsbQ directly interact within the PS II complex.

PsbQ may also be required for efficient PS II-LHC II supercomplex formation. Arabidopsis T-DNA mutants have been used to examine the extrinsic proteins of PS II (Allahverdiyeva et al. 2013). The psbqlpsbq2 mutant, which completely lacked PsbQ, exhibited a number of interesting phenotypes. This mutant accumulated only about $50 \%$ of the PS II-LHC II supercomplex observed to accumulate in the wild-type strain. Additionally, it exhibited very rapid state 1 to state 2 and rapid state 2 to state 1 transitions when compared to wild type. These occurred even though this strain exhibited low levels of LHC II phosphorylation. It is unclear if these functional defects are directly or indirectly associated with the lower accumulation of PS II-LHC II supercomplexes in this strain.
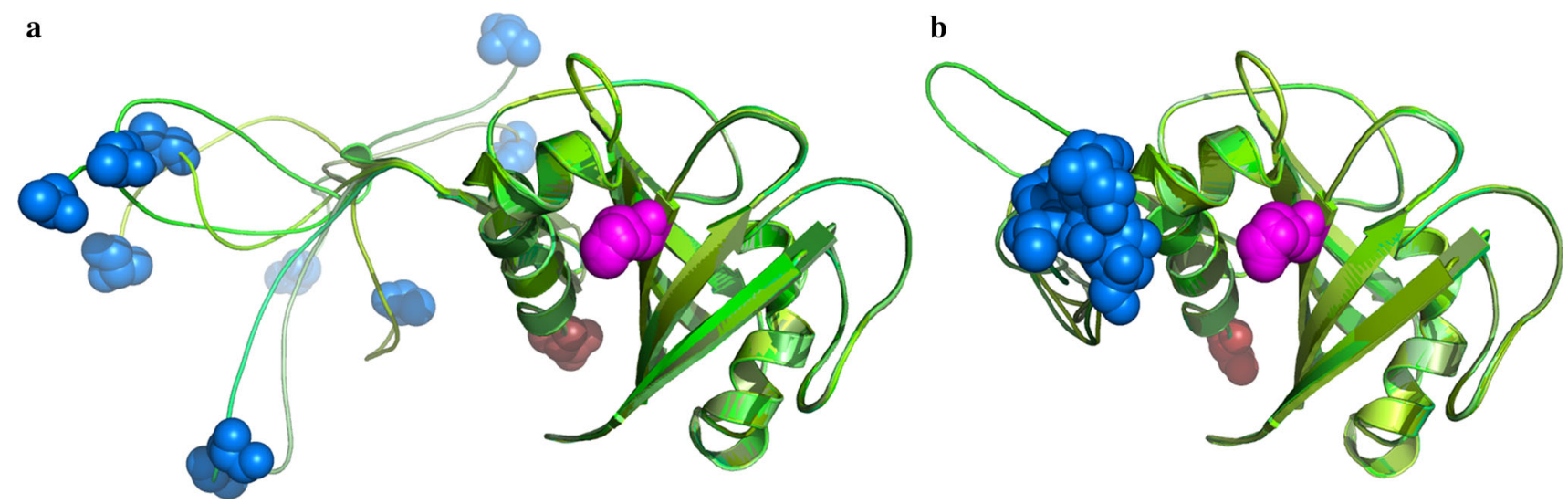

Fig. 4 Organization of the N-terminal Domain of PsbP when Bound to PS II. Molecular dynamic refinements of the N-terminal residues of PsbP, a without distance constraints and $\mathbf{b}$ with distance constraints provided by intramolecular BS3 crosslinking of PsbP when it is associated with PS II membranes (Mummadisetti et al. 2014) and using the 4RTI structure of PsbP (Cao et al. 2015). Shown are the ten lowest energy structures from the molecular dynamics structural refinement (Eswar et al. 2007). The $\mathrm{N}$-terminal residue ${ }^{1} \mathrm{~A}$ is shown as blue spheres and the $\mathrm{C}$-terminal residue ${ }^{186} \mathrm{~A}$ is shown as red spheres. Residue ${ }^{144} \mathrm{H}$ is shown as magenta spheres. In the ten models, the distance between ${ }^{1} \mathrm{~A}$ and ${ }^{144} \mathrm{H}$ was $9-18 \AA$ 
Two crystal structures for PsbQ are currently available (PDB:1NZE, (Calderone et al. 2003) and 1VYK, (Balsera et al. 2005)). While the overall fold of the protein is nearly identical in these two structures, significant differences exist in the structures of the N-termini. 1NZE lacks a resolved $\mathrm{N}$-terminus $\left({ }^{38} \mathrm{~F}\right.$ is the first identified residue) while a loop domain $\left({ }^{14} \mathrm{~S}-{ }^{33} \mathrm{Y}\right)$ is unresolved in the $1 \mathrm{VYK}$ structure. The $1 \mathrm{VYK}$ structure contains a short parallel $\beta$ sheet in the N-terminal domain, ${ }^{1} \mathrm{~A}-{ }^{44} \mathrm{P}$. Recently, an NMR solution structure for PsbQ [PDB:2MWQ, (Rathner et al. 2015)] has been presented which lacks this $\beta$-sheet feature and contains a short $\alpha$ helix $\left({ }^{36} \mathrm{D}-{ }^{41} \mathrm{Q}\right)$ in the same region. Unfortunately, residues ${ }^{1} \mathrm{~A}-{ }^{35} \mathrm{~K}$ appear unorganized in this structure. It is unclear, at this time, which of these latter structures best represents the organization of PsbQ in its unbound state. As with PsbP, significant conformational changes could occur upon binding to the photosystem.

Since no crystal structures are available for higher plant PS II, the locations of PsbP and PsbQ within the photosystem remain unclear. Two groups have used protein crosslinking coupled with tandem mass spectrometry to examine the location of PsbP in higher plant PS II membranes and its interaction with other PS II components. Ido et al. (Ido et al. 2014), using the zero-length protein crosslinker EDC, confirmed the interaction of PsbP: ${ }^{1} \mathrm{~A}$ PsbE: ${ }^{57} \mathrm{E}$, which had been described earlier (Ido et al. 2012). Additionally, a crosslinking interaction between PsbP: ${ }^{27} \mathrm{~K}$ and PsbR: $:{ }^{22} \mathrm{D}$ was identified. A novel tertiary crosslinked product involving two PsbP peptides and a single CP 26 peptide was also observed (PsbP: ${ }^{115} \mathrm{E}-$ PsbP: ${ }^{173} \mathrm{~K}-\mathrm{PsbP}:{ }^{174} \mathrm{~K}-\mathrm{CP} 26:{ }^{96} \mathrm{E}$ ). These results appear to indicate that PsbP is in van der Waals contact with PsbE, PsbR and CP 26. A model was presented which showed the association of these components with higher plant PS II. This model, which incorporates the 2VU4 structure (Kopecky et al. 2012) of PsbP, requires that the N-terminal fifteen amino acids of PsbP be highly extended ( $>40 \AA$ ), since the proposed location of PsbR occupies a position between PsbP and PsbE within the complex. Additionally, while PsbP and PsbQ were proposed to directly interact, no crosslinked residues were identified. Neither protein is in direct contact with PsbO, while both interact with CP 26 at the lumenal membrane surface. In Fig. 5a we have rendered this model including only the core subunits of PS II and the extrinsic proteins.

Our laboratory has also examined the interaction of PsbP and PsbQ with PS II membranes using the protein crosslinker BS3 (11.4 $\AA$ spacer arm). Radiolytic footprinting was used to examine the domains on these proteins exposed to the bulk solvent (Mummadisetti et al. 2014). As noted above, the $\mathrm{N}$-terminal domain $\left({ }^{1} \mathrm{~A}-{ }^{40} \mathrm{~K}\right)$ and $\mathrm{C}$-terminal domain $\left({ }^{170} \mathrm{~K}-{ }^{186} \mathrm{~A}\right)$ appear closely associated. PsbP appears to form a compact structure when bound to the PS
II membrane and does not exhibit a long $\mathrm{N}$-terminal extension. Additionally, two independent crosslinked products between PsbP and PsbQ were identified (PsbP: $:{ }^{93} \mathrm{Y}$ and PsbP: ${ }^{96} \mathrm{~K}$ were both crosslinked to PsbQ: ${ }^{1} \mathrm{E}$ ), and intramolecular crosslinked products were observed within PsbQ (PsbQ: $:{ }^{98} \mathrm{~K}$ and PsbQ: ${ }^{101} \mathrm{~K}$ can both be crosslinked to PsbQ: ${ }^{133} \mathrm{Y}$ ). We presented two models for the association of PsbP and PsbQ with PS II that cannot be differentiated at this time. In Fig. 5b, c we have updated these models to include the 4HTI structure (Cao et al. 2015) for PsbP. This is critically important since both PsbP: ${ }^{93} \mathrm{Y}$ and PsbP: ${ }^{96} \mathrm{~K}$ reside in the loop of PsbP, which was not resolved in previous structures. In Fig. 5b the position of PsbQ is analogous to that proposed for CyanoQ in cyanobacteria (Liu et al. 2014). These authors did not examine the position of CyanoP within the photosystem. In Fig. 5c, the location of PsbQ is consistent with the location of PsbQ' in the crystal structure of the red algal ${ }^{2}$ Cyanidium caldarium PS II (Shen, J.-R., 16th International Congress on Photosynthesis, Plenary Lecture (2013), personal communication). PsbP has not been identified in the red alga.

Additionally, we observed several intramolecular crosslinked residues in PsbQ. PsbQ: ${ }^{98} \mathrm{~K}$ and PsbQ: ${ }^{101} \mathrm{~K}$ can both be crosslinked to PsbQ: ${ }^{133} \mathrm{Y}$ even though these residues are separated by $>30 \AA$. This is highly reminiscent of the proposal in cyanobacterial PS II that CyanoQ is present as an antiparallel homodimer (Liu et al. 2014). Our observation of an apparent dimer of PsbQ subunits would seem to favor an organization of PsbQ similar to that proposed for CyanoQ (Liu et al. 2014), positioning the PsbQ dimer at the PS II dimer interface. However, an alternative possibility is that the observed putative PsbQ dimer bridges adjacent PS II core dimers. This might be possible in higher plant PS II membranes which exhibit pseudocrystalline arrays of PS II (Boekema et al. 2000) and could be consistent with the location of PsbQ' in the crystal structure of the red algal Cyanidium caldarium PS II (Shen, J.-R., 16th International Congress on Photosynthesis, Plenary Lecture (2013), personal communication).

\section{The PsbR protein of green algae and higher plants}

PsbR, which remains the most enigmatic extrinsic protein in higher plant PS II, has recently been the object of some additional scrutiny. As discussed in our earlier review (Bricker et al. 2012), PsbR has been implicated in the association of PsbP with the photosystem. While the threedimensional structure of PsbR is unknown, I-TASSER (Zhang 2008) models for this component have been presented (Bricker et al. 2012; Ido et al. 2014), these must,

\footnotetext{
${ }^{2}$ A structure (PDB: 4YUU) has been deposited in the Protein Data Bank and, and is currently on hold pending publication.
} 

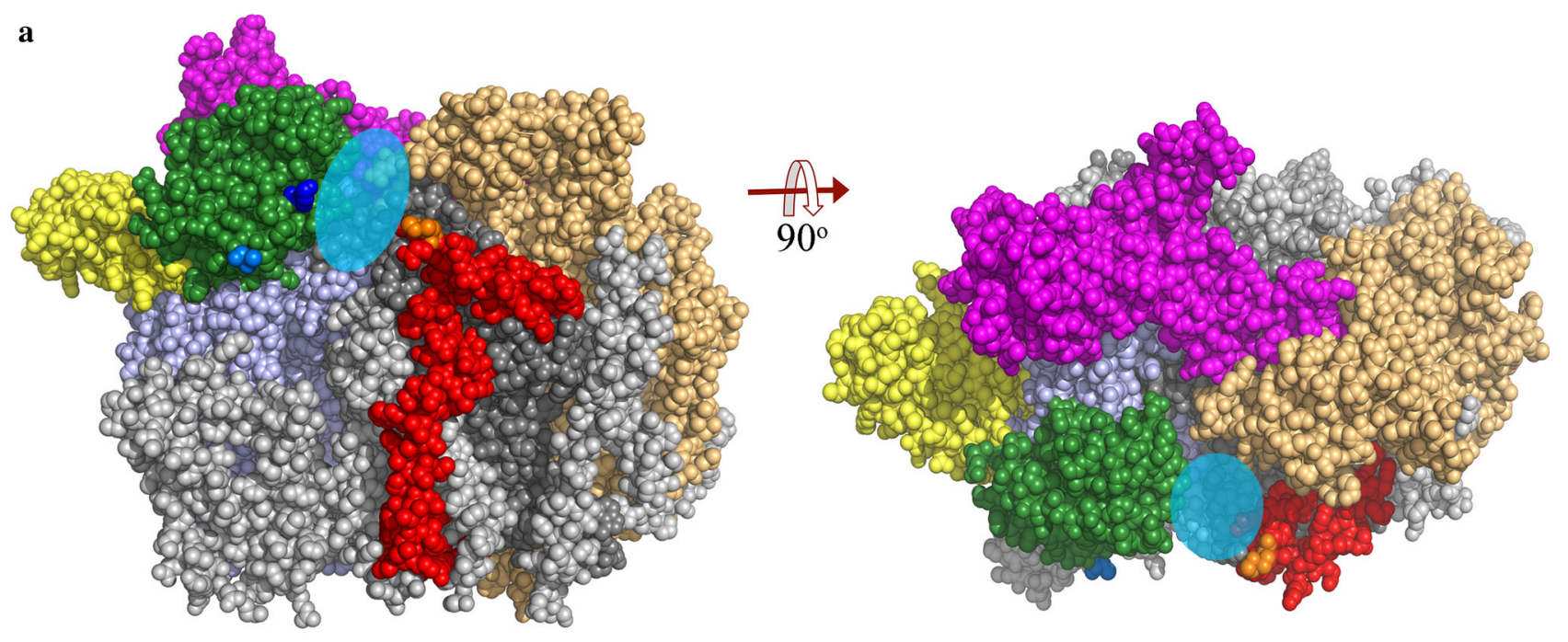

b
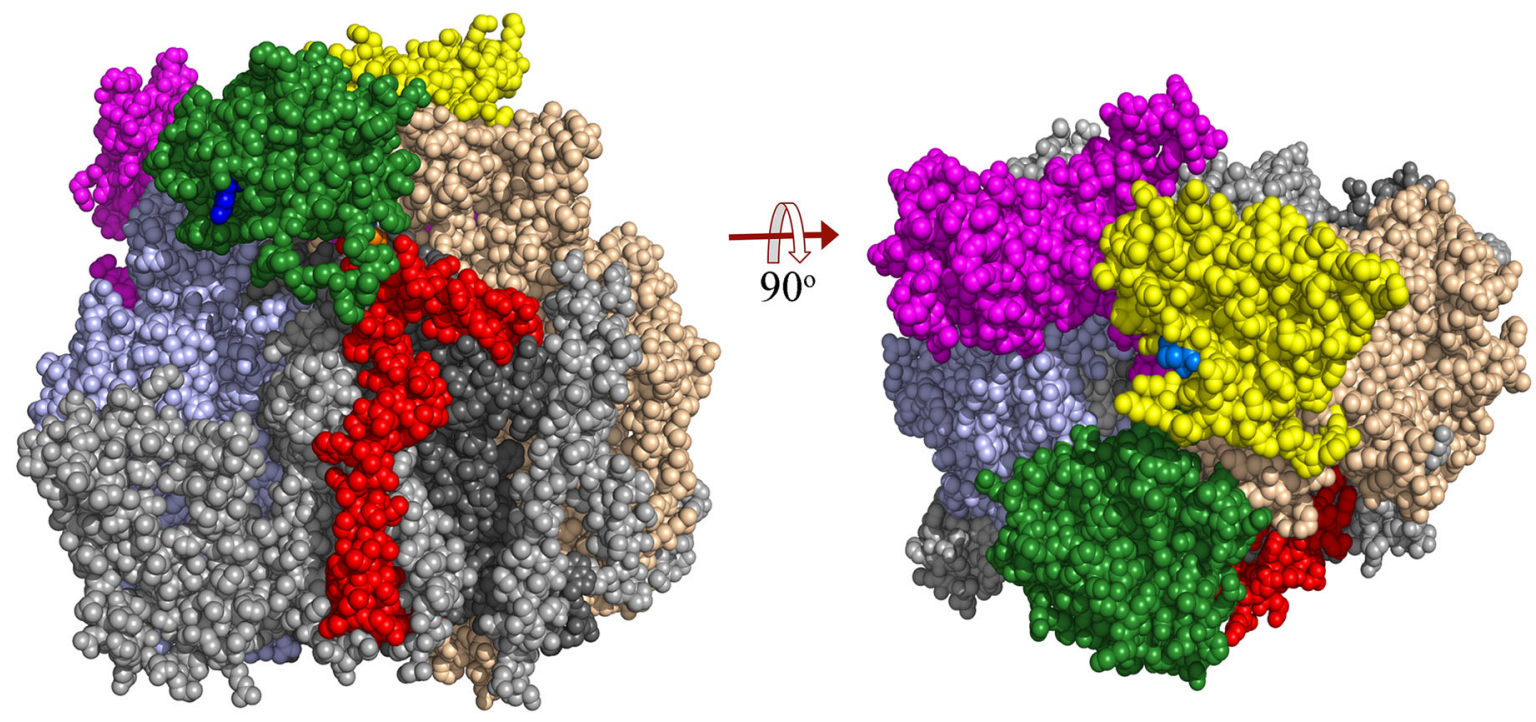

c
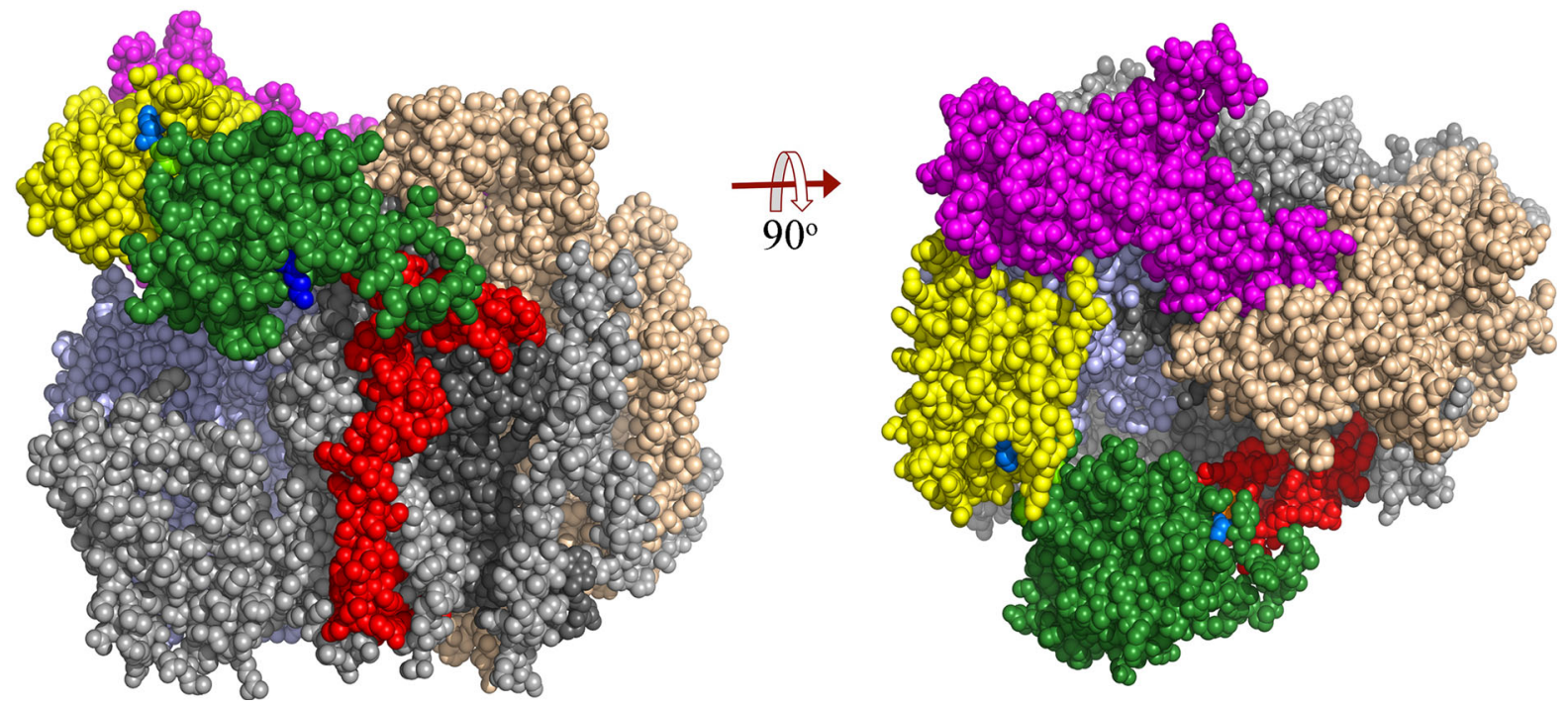
4 Fig. 5 Proposed models for the interaction of PsbP and PsbQ with Higher Plant PS II Based on Protein Crosslinking Studies. The extrinsic proteins PsbP, PsbQ and PsbO are shown as green, yellow, and magenta spheres, respectively. The intrinsic proteins PsbC, PsbB, and PsbE are shown as pale blue, tan, and red spheres, respectively. a left panel, our rendering of the model presented by Ido et al. (2014) which is based on the cyanobacterial PS II structure (Umena et al. 2011), the 1V2B structure of Nicotiana PsbP (Ifuku et al. 2004) and the 1NZE structure of spinach PsbQ (Calderone et al. 2003). The proposed location of PsbR is shown as a cyan ellipse as no actual crystal structure is currently available. In this panel, $P \operatorname{sbP}:{ }^{27} \mathrm{~K}$ is shown as blue spheres and is EDC-crosslinked to PsbR: ${ }^{22} \mathrm{D}$ (not shown). The first resolved N-terminal residue of PsbP in the 1V2B structure $\left(\mathrm{PsbP}:{ }^{16} \mathrm{~T}\right)$ is shown as light blue spheres. The PsbE residue ${ }^{59} \mathrm{E}$, which is the equivalent of ${ }^{57} \mathrm{E}$ in higher plant $\mathrm{PsbE}$, interacts with PsbP: ${ }^{1} \mathrm{~A}$ (Ido et al. 2012) and is shown in orange. This view is within the plane of the membrane looking towards one PS II monomer. a right panel, this view is looking from the lumen towards the surface of the PS II monomer. b left panel, the first of two updated models based on the data presented by Mummadisetti et al. (2014). These utilize the cyanobacterial PS II structure (Umena et al. 2011), the updated 4RTI structure of spinach PsbP (Cao et al. 2015), including the N-terminal crosslinking constraints described in Mummadisetti et al. (2014), and the 1VYK structure of spinach PsbQ (Balsera et al. 2005). In this panel, PsbP: ${ }^{27} \mathrm{~K}$ is shown as blue spheres and is crosslinked to PsbR: ${ }^{22} \mathrm{D}$ (not shown). The location of the N-terminus of PsbP $\left({ }^{1} \mathrm{~A}\right)$, which was based on molecular dynamic refinement using BS3 crosslinking distance constraints (Fig. 2) and is hidden in this view, was modeled to be in van der Waals contact with the PsbE residue ${ }^{57} \mathrm{E}$ which is shown in orange (barely visible, equivalent to ${ }^{59} \mathrm{E}$ in $T$. vulcanus). The position of PsbQ was modeled after the proposed location of CyanoQ (Liu et al. 2014). This view is within the plane of the membrane looking towards one PS II monomer. b right panel, this view is looking from the lumen towards the surface of the PS II monomer. The residue PsbQ: ${ }^{1} \mathrm{E}$, which is crosslinked to residues PsbP: $:{ }^{93} \mathrm{Y}$ and PsbP: $:{ }^{96} \mathrm{~K}$ (hidden from view) with the crosslinker BS3, is shown in light blue. $\mathbf{c}$ left panel, the second of two updated models based on those presented by Mummadisetti et al. (2014). The structures used to generate this model are as in $\mathbf{b}$, above. In this panel, PsbP: ${ }^{27} \mathrm{~K}$ is shown as blue spheres and is crosslinked to PsbR: ${ }^{22} \mathrm{D}$ (not shown). The interaction of PsbP: ${ }^{1} \mathrm{~A}$ with PsbE: ${ }^{59} \mathrm{E}$, is hidden in this view. The interaction of PsbQ: ${ }^{1} \mathrm{E}$ which is crosslinked to residues PsbP: ${ }^{93} \mathrm{Y}$ and PsbP: ${ }^{96} \mathrm{~K}$ is also hidden. PsbP: ${ }^{27} \mathrm{~K}$ is shown as blue spheres and is crosslinked to PsbR: ${ }^{22} \mathrm{D}$ (not shown). c right panel, this view is looking from the lumen towards the surface of the PS II monomer. PsbP: ${ }^{1} \mathrm{~A}$ is shown as light blue spheres and is in van der Waals contact with PsbE: ${ }^{57} \mathrm{E}$ (equivalent to ${ }^{59} \mathrm{E}$ in $T$. vulcanus), shown as orange spheres and barely visible in this view. The residue PsbQ: ${ }^{1} \mathrm{E}$ is shown in light blue and is crosslinked to residues PsbP: ${ }^{93} \mathrm{Y}$ and PsbP: ${ }^{96} \mathrm{~K}$ which are shown in light green (barely visible). This model is consistent with the location of PsbQ' in the crystal structure of the red algal ${ }^{2}$ Cyanidium caldarium PS II (Shen, J.-R., 16th International Congress on Photosynthesis, Plenary Lecture (2013), personal communication)

however, be considered highly speculative. It is even unclear if PsbR is an extrinsic protein, as originally reported (Ljungberg et al. 1984), or an intrinsic PS II component, anchored by an unusual C-terminal domain transmembrane $\alpha$-helix (Webber et al. 1989). While the structure of PsbR has not been determined, its location with respect to PsbP has been identified. Using protein crosslinking with EDC and tandem mass spectrometry, Ido et al. (2014) determined that PsbP: ${ }^{27} \mathrm{~K}$ is in van der Waals contact PsbR: ${ }^{22} \mathrm{D}$. These authors presented a model for the location of PsbR with the photosystem in which PsbR was positioned between PsbP and PsbE (Fig. 6a). This model requires that $\mathrm{PsbP}$ have an extended $\mathrm{N}$-terminal domain. As noted above, PS II-bound PsbP exhibits a compact structure and its N-terminus does not appear to be highly extended (Mummadisetti et al. 2014). Additionally, using oxidative footprinting, these authors identified a domain on PsbP which was adjacent to PsbP: ${ }^{27} \mathrm{~K}$ and was shielded from the bulk solvent. It was suggested that this domain represented the interaction site of PsbP with PsbR. In Fig. 6 we present two models for the location of PsbR. The first (Fig. 6b) is based on a model consistent with locating PsbQ at the PS II dimer interface (i.e. in a location analogous to that proposed for CyanoQ (Liu et al. (2014)). The second (Fig. 6c) is consistent with the location of CyanoQ' in the red alga Cyanidium caldarium ${ }^{2}$ PS II (Shen, J.-R., 16th International Congress on Photosynthesis, Plenary Lecture (2013), personal communication). The models presented in Fig. 6 are speculative but based on the protein crosslinking information provided by Ido et al. (2014) and Mummadisetti et al. (2014).

In Arabidopsis, T-DNA lines have been used to investigate the role of PsbR and the other extrinsic proteins in the photosystem (Allahverdiyeva et al. 2013). As expected, the $p s b r$ mutant exhibited severe reductions in PsbP (3\% wild-type levels and PsbQ (18\% wild-type levels). Surprisingly, these plants were photoautotrophic, whereas their psbpl mutant plants were not. As noted above, these authors suggested that PsbP was required only during the early stages of plant development and that the small amount of PsbP present in the $p s b r$ mutant was sufficient to support the development of photoautotrophy. The psbr mutant also exhibited a decrease in the accumulation of PS II-LHC II supercomplexes. It was unclear if this was directly the result of the loss of PsbR or if this was a secondary effect due to the low amount of PsbQ present also observed in this strain. The psbqlpsbq2 double mutant exhibited an even lower steady-state accumulation of PS II-LHC II supercomplex than did $p s b r$ plants. Interestingly, the $p s b r$ mutant, as well as the psbqlpsbq2 and psbqlps$b q 2 p s b r$ mutants, exhibited a very rapid state 1 to state 2 transition when compared to wild type, even though these mutants exhibited significantly lower levels of LHC II phosphorylation. They also exhibited a more rapid state 2 to state 1 transition. It should be noted that the rate of phosphorylation and dephosphorylation during the state transitions were not examined. Clearly, these results indicate that the interplay between this cohort of proteins is quite complex.

In Chlamydomonas, LHCSR3 is required for the development of the $\mathrm{qE}$ component of non-photochemical 


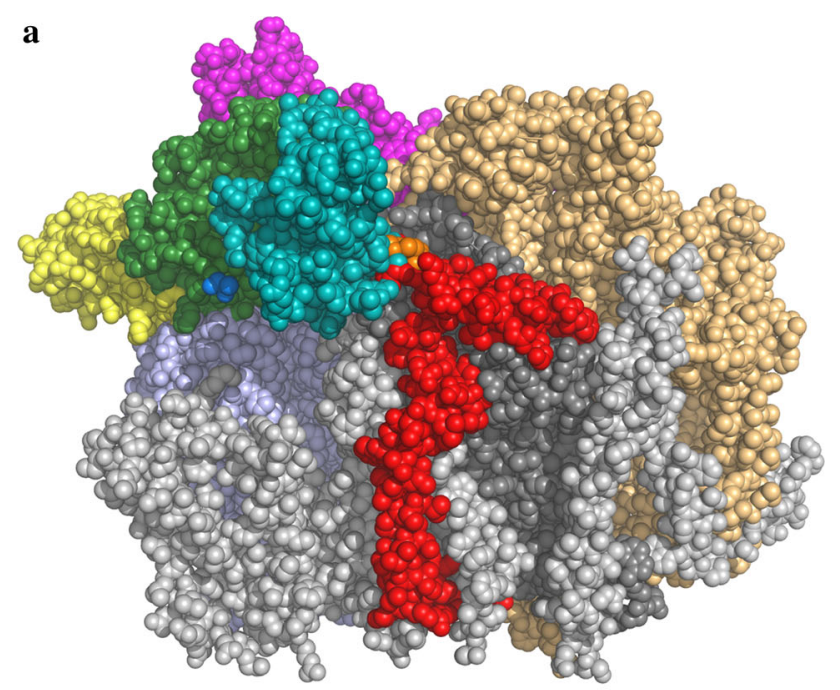

b

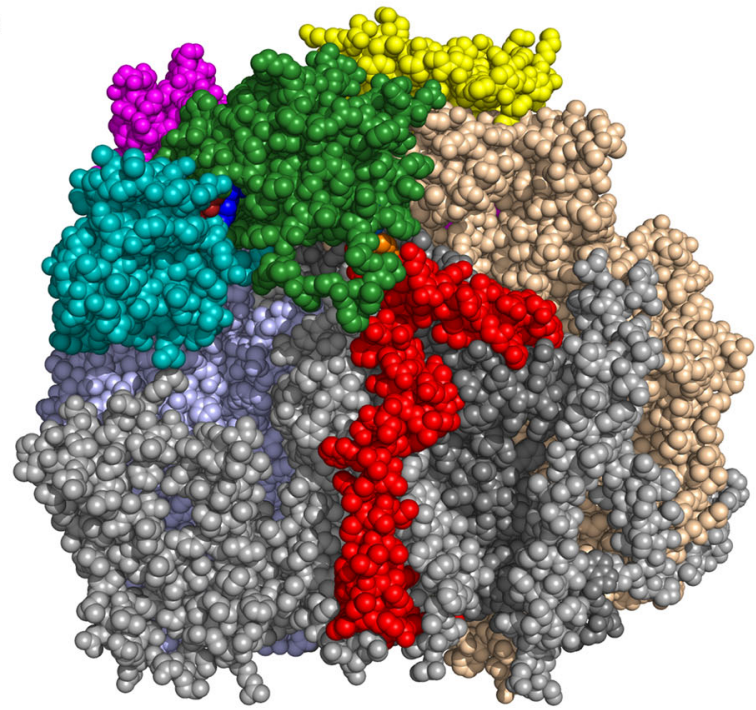

c

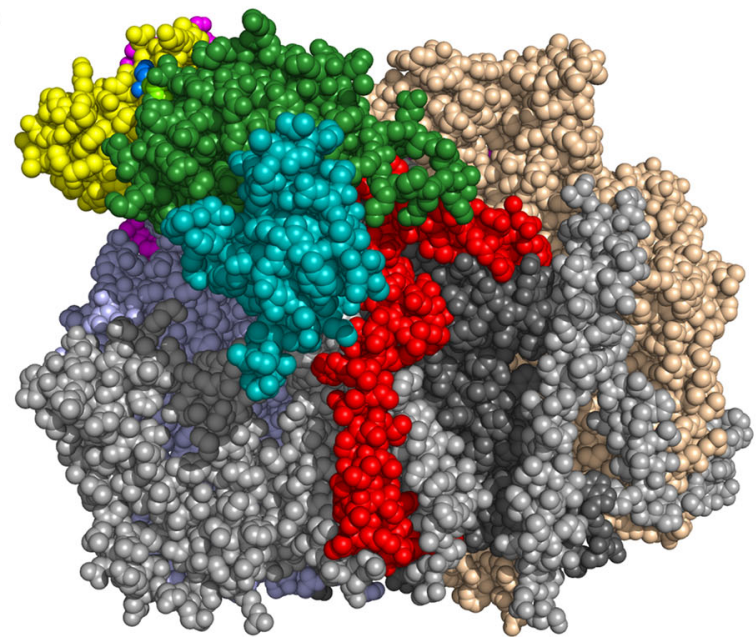

4Fig. 6 Proposed models for the location of PsbR with Higher Plant PS II Based on Protein Crosslinking Studies. The extrinsic proteins PsbR, PsbP, PsbQ, and PsbO are shown as cyan, green, yellow, and magenta spheres, respectively. The intrinsic proteins PsbC, PsbB, and PsbE are shown as pale blue, tan, and red spheres, respectively. The hypothetical PsbR structure shown is an I-TASSER model (Zhang 2008) and should be viewed with skepticism. This model did not incorporate the hypothetical C-terminal $\alpha$-helix proposed by Webber et al. (1989). a our rendering of the model presented by Ido et al. (2014) in Fig. 5a, above, showing the location of PsbR between PsbP and PsbE. b Hypothetical position of PsbR in Fig. 5b, above. The position of PsbQ was modeled after the proposed location of CyanoQ (Liu et al. 2014). c, Hypothetical position of PsbR in Fig. 5c, above. The position of PsbQ is consistent with the location of PsbQ' in the crystal structure of the red algal ${ }^{2}$ Cyanidium caldarium PS II (Shen, J.-R., 16th International Congress on Photosynthesis, Plenary Lecture (2013), personal communication)

quenching (NPQ) in the green algae (Peers et al. 2009) and functionally associates with the PS II-LHC II supercomplex (Tokutsu and Minagawa 2013). PsbR also appears to be required for the binding of LHCSR3 to the PS II-LHC II supercomplex (Xue et al. 2015). Down regulation of PsbR led to the decrease in NPQ development and a marked decrease in the amount of LHCSR3 associated with the supercomplex. Consistent with earlier studies, the down regulation of PsbR also destabilized the association of PsbP with the PS II-LHC II supercomplex. The absence of PsbP and PsbQ in the FUD39 mutant (Mayfield et al. 1987) did not lead to the loss of PsbR or LHCSR3 from the supercomplex, however the total amount of PS II-LHC II was markedly lower in this strain. The authors argued that the binding of LHCSR3 to PsbR allowed for direct energetic coupling between LHCSR3 and the PS II core and its associated light-harvesting components, leading to efficient energy dissipation. The observation of EDC-crosslinked products between PsbR and CP 26 (Ido et al. 2014) supports the hypothesis that PsbR is associated with lightharvesting components of PS II.

\section{Conclusions}

Clearly, over the last 3 years significant progress has been made in our understanding of the structures and functions of these the extrinsic proteins of PS II. Molecular dynamic modeling of the extended hydrogen-bonding networks at the interface of the extrinsic and intrinsic proteins is playing an important role in elucidating proton exit and water uptake pathways. Additionally, such studies may assist in identifying binding determinants for the extrinsic proteins to the photosystem. Difference FTIR has provided the first hints of possible mechanisms by which the extrinsic proteins modulate the inorganic cofactor requirements of the photosystem. The use of alternative 
structural methods, including protein crosslinking coupled with tandem mass spectrometry and surface plasmon resonance techniques, have provided our first glimpses into the possible organization of CyanoQ and CyanoP within cyanobacterial PS II and PsbP, PsbQ and PsbR in the higher plant photosystem. These and many other studies will form the basis for future advances in this field.

Author contribution statement J.R., L.F., M.M. and T.B. substantially contributed to this manuscript and were involved in the drafting, preparation of models and critical revision. All authors provide final approval of the version to be published and are accountable for all aspects of the work.

Acknowledgments This work was supported by the Division of Chemical Sciences, Geosciences, and Biosciences, Office of Basic Energy Sciences of the U.S. Department of Energy through Grant DEFG02-98ER20310 to T.B. and L.F.

\section{Compliance with ethical standards}

Conflict of interest The authors declare no conflict of interests.

\section{References}

Abasova L, Deak Z, Schwarz R, Vass I (2011) The role of the PsbU subunit in the light sensitivity of PSII in the cyanobacterium Synechococcus 7942. J Photochem Photobiol B 105:149-156

Allahverdiyeva Y, Mamedov F, Holmström M, Nurmi M, Lundin B, Styring S, Spetea C, Aro E-M (2009) Comparison of the electron transport properties of the psbol and psbo2 mutants of Arabidopsis thaliana. Biochim Biophys Acta 1787:1230-1237

Allahverdiyeva Y, Suorsa M, Rossi F, Pavesi A, Kater M, Antonacci A, Tadini L, Pribil M, Schneider A, Wanner G, Leister D, Aro E-M, Barbato R, Pesaresi P (2013) Arabidopsis plants lacking PsbQ and PsbR subunits of the oxygen-evolving complex show altered PSII super-complex organization and short-term adaptive mechanisms. Plant J 75:671-684

Aoi M, Kashino Y, Ifuku K (2014) Function and association of CyanoP in photosystem II of Synechocystis sp. PCC 6803. Res Chem Intermed 40:3209-3217

Balint I, Bhattacharya J, Perelman A, Schatz D, Moskovitz Y, Keren N, Schwarz R (2006) Inactivation of the extrinsic subunit of photosystem II, PsbU, in Synechococcus PCC 7942 results in elevated resistance to oxidative stress. FEBS Lett 580:2117-2122

Balsera M, Arellano JB, Revuelta JL, de las Rivas J, Hermoso JA (2005) The 1.49 A resolution crystal structure of PsbQ from photosystem II of Spinacia oleracea reveals a PPII structure in the N-terminal region. J Mol Biol 350:1051-1060

Boekema EJ, van Breemen JF, van Roon H, Dekker JP (2000) Arrangement of photosystem II supercomplexes in crystalline macrodomains within the thylakoid membrane of green plant chloroplasts. J Mol Biol 301:1123-1133

Bondar A-N, Dau H (2012) Extended protein/water H-bond networks in photosynthetic water oxidation. Biochim Biophys Acta 1817:1177-1190

Bondarava N, Krieger-Liszkay A (2007) Manganese binding to the $23 \mathrm{kDa}$ extrinsic protein of photosystem II. Biochim Biophys Acta 1767:583-588
Bondarava N, Beyer P, Krieger-Liszkay A (2005) Function of the $23 \mathrm{kDa}$ extrinsic protein of Photosystem II as a manganese binding protein and its role in photoactivation. Biochim Biophys Acta 1708:63-70

Bricker TM (1992) Oxygen evolution in the absence of the $33 \mathrm{kDa}$ manganese-stabilizing protein. Biochemistry 31:4623-4628

Bricker TM, Burnap RL (2005) The extrinsic proteins of Photosystem II. In: Wydrzynski T, Satoh K (eds) Photosystem II: the water/plastoquinone oxido-reductase of photosynthesis. Springer, Dordrecht, pp 95-120

Bricker TM, Frankel LK (1998) The structure and function of the $33 \mathrm{kDa}$ extrinsic protein of photosystem II. A critical review. Photosyn Res 56:157-173

Bricker TM, Frankel LK (2008) The Arabidopsis psbol mutant cannot efficiently utilize calcium in support of photosynthetic oxygen evolution. J Biol Chem 283:29022-29027

Bricker TM, Frankel LK (2011) Auxiliary functions of the PsbO, PsbP and PsbQ proteins of higher plant photosystem II: a critical analysis. J Photochem Photobiol B: Biol 104:165-178

Bricker TM, Young A, Frankel LK, Putnam-Evans C (2002) Introduction of the 305Arg $\rightarrow$ 305Ser mutation in the large extrinsic loop E of the CP43 protein of Synechocystis sp. PCC 6803 leads to the loss of cytochrome $\mathrm{c}_{550}$ binding to photosystem II. Biochim Biophys Acta 1556:92-96

Bricker TM, Roose JL, Fagerlund RD, Frankel LK, Eaton-Rye JJ (2012) The extrinsic proteins of photosystem II. Biochim Biophys Acta 1817:121-142

Bricker TM, Roose JL, Zhang P, Frankel LK (2013) The PsbP family of proteins. Photosynth Res 116:235-250

Bricker TM, Mummadisetti MP, Frankel LK (2015) Recent advances in the use of mass spectrometry to examine structure/function relationships in photosystem II. J Photochem Photobiol B Biol 152:227-246

Burch BD, Bricker TM, Putnam-Evans C (2012) Mutations in the CP43 protein of photosystem II affect PSII function and cytochrome $c_{550}$ binding. In: Najafpour MM (ed) Atrificial photosynthesis. InTech, Rijeka, pp 53-78

Calderone V, Trabucco M, Vujicic A, Battistutta R, Giacometti GM, Andreucci F, Barbato R, Zanotti G (2003) Crystal structure of the PsbQ protein of photosystem II from higher plants. EMBO Rep 4:900-905

Cao P, Xie Y, Li M, Pan X, Zhang H, Zhao X, Su X, Cheng T, Chang W (2015) Crystal structure analysis of extrinsic PsbP protein of photosystem II reveals a manganese-induced conformational change. Mol Plant 8:664-666

Cardona T, Sedoud A, Cox N, Rutherford AW (2012) Charge separation in photosystem II: a comparitive and evolutionary approach. Biochim Biophys Acta 1817:26-43

Cole J, Boska M, Blough NV, Sauer K (1986) Reversible and irreversible effects of alkaline $\mathrm{pH}$ on Photosystem II electrontransfer reactions. Biochim Biophys Acta BBA Bioenerg 848:41-47

Cormann KU, Bartsch M, Rogner M, Nowaczyk MM (2014) Localization of the CyanoP binding site on photosystem II by surface plasmon resonance spectroscopy. Front Plant Sci 5:595

Duchoslav M, Fischer L (2015) Parallel subfunctionalisation of PsbO protein isoforms in angiosperms revealed by phylogenetic analysis and mapping of sequence variability onto protein structure. BMC Plant Biol 15:133

Eaton-Rye JJ, Shand JA, Nicoll WS (2003) pH-dependent photoautotrophic growth of specific photosystem II mutants lacking lumenal extrinsic polypeptides in Synechocystis sp. PCC 6803. FEBS Lett 543:148-153

Eswar N, Webb B, Marti-Renom MA, Madhusudhan MS, Eramian D, Shen M-Y, Pieper U, Sali A (2007) Comparative protein 
structure modeling using MODELLER. Curr Protoc Prot Sci 50:2.9.1-2.9.31

Fagerlund RD, Eaton-Rye JJ (2011) The lipoproteins of cyanobacterial photosystem II. J Photochem Photobiol B 104:191-203

Frankel LK, Cruz JA, Bricker TM (1999) Carboxylate groups on the manganese-stabilizing protein are required for its efficient binding to photosystem II. Biochemistry 38:14271-14278

Galetskiy D, Lohscheider JN, Kononikhin AS, Popov IA, Nikolaev EN, Adamska I (2011) Mass spectrometric characterization of photooxidative protein modifications in Arabidopsis thaliana thylakoid membranes. Rapid Commun Mass Spectrom 25:184-190

Guerrero F, Sedoud A, Kirilovsky D, Rutherford AW, Ortega JM, Roncel M (2011) A high redox potential form of cytochrome $\mathrm{c}_{550}$ in photosystem II from Thermosynechococcus elongatus. J Biol Chem 286:5985-5994

Ido K, Ifuku K, Yamamoto Y, Ishihara S, Murakami A, Takabe K, Miyake C, Sato F (2009) Knockdown of PsbP protein does not prevent assembly of the dimeric PSII core complex but impairs accumulation of Photosystem II supercomplexes in tobacco. Biochim Biophys Acta 1787:873-881

Ido K, Kakiuchi S, Uno C, Nishimura T, Fukao Y, Noguchi T, Sato F, Ifuku K (2012) The conserved His-144 in the PsbP protein is important for the interaction between the PsbP N-terminus and the Cyt $b_{559}$ subunit of Photosystem II. J Biol Chem 287:26377-26387

Ido K, Nield J, Fukao Y, Nishimura T, Sato F, Ifuku K (2014) Crosslinking evidence for multiple interactions of the PsbP and PsbQ proteins in a higher plant photosystem II supercomplex. J Biol Chem 289:20150-20157

Ifuku K (2014) The PsbP and PsbQ family proteins in the photosynthetic machinery of chloroplasts. Plant Physiol Biochem 81:108-114

Ifuku K, Sato F (2001) Importance of the N-terminal sequence of the extrinsic $23 \mathrm{kDa}$ polypeptide in photosystem II in ion retention in oxygen evolution. Biochim Biophys Acta 1546:196-204

Ifuku K, Sato F (2002) A truncated mutant of the extrinsic 23-kDa protein that absolutely requires the extrinsic $17-\mathrm{kDa}$ protein for $\mathrm{Ca}^{2+}$ retention in photosystem II. Plant Cell Physiol 43:1244-1249

Ifuku K, Nakatsu T, Kato H, Sato F (2004) Crystal structure of the PsbP protein of photosystem II from Nicotiana tabacum. EMBO Rep 5:362-367

Ifuku K, Yamamoto J, Ono T-A, Ishihara S, Sato F (2005) PsbP protein, but not PsbQ protein, is essential for the regulation and stabilization of photosystem II in higher plants. Plant Physiol 139:1175-1184

Ifuku K, Ishihara S, Shimamoto R, Ido K, Sato F (2008) Structure, function, and evolution of the PsbP protein family in higher plants. Photosyn Res 98:427-437

Inoue-Kashino N, Kashino Y, Satoh K, Terashima I, Pakrasi HB (2005) PsbU provides a stable architecture for the oxygenevolving system in cyanobacterial photosystem II. Biochemistry 44:12214-12228

Ischiropoulos H (2009) Protein tyrosine nitration-an update. Arch Biochem Biophys 484:117-121

Ishikawa Y, Schroder WP, Funk C (2005) Functional analysis of the PsbP-like protein (sll1418) in Synechocystis sp. PCC 6803. Photosynth Res 84:257-262

Jackson SA, Eaton-Rye JJ (2015) Characterization of a Synechocystis sp. PCC 6803 double mutant lacking the CyanoP and Ycf48 proteins of Photosystem II. Photosynth Res 124:217-229

Jackson SA, Fagerlund RD, Wilbanks SM, Eaton-Rye JJ (2010) Crystal structure of PsbQ from Synechocystis sp. PCC 6803 at $1.8 \AA$ : implications for binding and function in cyanobacterial photosystem II. Biochemistry 49:2765-2767
Jackson SA, Hinds MG, Eaton-Rye JJ (2012) Solution structure of CyanoP from Synechocystis sp. PCC 6803: new insights on the structural basis for functional specialization amongst PsbP family proteins. Biochim Biophys Acta 1817:1331-1338

Jackson SA, Hervey JR, Dale AJ, Eaton-Rye JJ (2014) Removal of both Ycf48 and Psb27 in Synechocystis sp. PCC 6803 disrupts photosystem II assembly and alters $\mathrm{Q}_{\mathrm{A}}^{-}$oxidation in the mature complex. FEBS Lett 588:3751-3760

Kakiuchi S, Uno C, Ido K, Nishimura T, Noguchi T, Ifuku K, Sato F (2012) The PsbQ protein stabilizes the functional binding of the PsbP protein to photosystem II in higher plants. Biochim Biophys Acta 1817:1346-1351

Komenda J, Nickelsen J, Tichy M, Prasil O, Eichacker LA, Nixon PJ (2008) The cyanobacterial homologue of HCF136/YCF48 is a component of an early photosystem II assembly complex and is important for both the efficient assembly and repair of photosystem II in Synechocystis sp. PCC 6803. J Biol Chem 283:22390-22399

Kopecky V, Kohoutova J, Lapkouski M, Hofbauerova K, Sovova Z, Ettrichova O, González-Pérez S, Dulebo A, Kaftan D, Smatanova IK, Revuelta JL, Arellano JB, Carey J, Ettrich R (2012) Raman spectroscopy adds complementary detail to the highresolution X-ray crystal structure of photosynthetic PsbP from Spinacia oleracea. PLoS One 7:e46694

Kuwabara T, Murata N (1982) Inactivation of photosynthetic oxygen evolution and concomitant release of three polypeptides in the photosystem II particles of spinach chloroplasts. Plant Cell Physiol 23:533-539

Leuschner C, Bricker TM (1996) Interaction of the $33 \mathrm{kDa}$ extrinsic protein with photosystem II:rebinding of the $33 \mathrm{kDa}$ extrinsic protein to photosystem II membranes which contain four, two, or zero manganese per photosystem II reaction center. Biochem 35:4551-4557

Liu H, Zhang H, Weisz DA, Vidavsky I, Gross ML, Pakrasi HB (2014) MS-based cross-linking analysis reveals the location of the PsbQ protein in cyanobacterial photosystem II. Proc Natl Acad Sci USA 111:4638-4643

Liu H, Weisz DA, Pakrasi HB (2015) Multiple copies of the PsbQ protein in a cyanobacterial photosystem II assembly intermediate complex. Photosynth Res 126:375-383

Ljungberg U, Akerlund H-E, Larsson C, Andersson B (1984) Identification of polypeptides associated with the 23 and $33 \mathrm{kDa}$ proteins of photosynthetic oxygen evolution. Biochim Biophys Acta 767:145-152

Lorch S, Capponi S, Pieront F, Bondar A-N (2015) Dynamic carboxylate/water networks on the surface of the PsbO Subunit of photosystem II. J Phys Chem B 119:12173-12181

Mayfield SP, Rahire M, Frank G, Zuber H, Rochaix JD (1987) Expression of the nuclear gene encoding oxygen-evolving enhancer protein 2 is required for high levels of photosynthetic oxygen evolution in Chlamydomonas reinhardtii. Proc Natl Acad Sci USA 84:749-753

Michoux F, Takasaka K, Boehm M, Nixon PJ, Murray JW (2010) Structure of CyanoP at $2.8 \AA$ : implications for the evolution and function of the PsbP subunit of Photosystem II. Biochemistry 49:7411-7413

Michoux F, Boehm M, Bialek W, Takasaka K, Maghlaoui K, Barber J, Murray JW, Nixon PJ (2014) Crystal structure of CyanoQ from the thermophilic cyanobacterium Thermosynechococcus elongatus and detection in isolated photosystem II complexes. Photosynth Res 122:57-67

Miyao M, Murata N (1985) The $\mathrm{Cl}^{-}$effect on photosynthetic oxygen evolution: interaction of $\mathrm{Cl}^{-}$with $18-\mathrm{kDa}, 24-\mathrm{kDa}$ and $33-\mathrm{kDa}$ proteins. FEBS Lett 180:303-308

Mummadisetti MP, Frankel LK, Bellamy H, Sallans L, Goettert JS, Brylinski M, Limbach PA, Bricker TM (2014) Use of protein 
cross-linking and radiolytic footprinting to elucidate PsbP and PsbQ interactions within higher plant Photosystem II. Proc Natl Acad Sci USA 111:16178-16183

Nagao R, Tomo T, Noguchi T (2015) Effects of extrinsic proteins on the protein conformation of the oxygen-evolving center in cyanobacterial photosystem II as revealed by Fourier transform infrared spectroscopy. Biochemistry 54:2022-2031

Nelson N, Yocum CF (2006) Structure and function of photosystems I and II. Ann Rev Plant Biol 57:521-565

Nishimura T, Uno C, Ido K, Nagao R, Noguchi T, Sato F, Ifuku K (2014) Identification of the basic amino acid residues on the PsbP protein involved in the electrostatic interaction with photosystem II. Biochim Biophys Acta 1837:1447-1453

Nishiyama Y, Los DA, Murata N (1999) PsbU, a protein associated with photosystem II, is required for the acquisition of cellular thermotolerance in Synechococcus species PCC 7002. Plant Physiol 120:301-308

Offenbacher AR, Polander BC, Barry BA (2013) An intrinsically disordered photosystem II subunit, PsbO, provides a structural template and a sensor of the hydrogen-bonding network in photosynthetic water oxidation. J Biol Chem 288:29056-29068

Peers G, Truong TB, Ostendorf E, Busch A, Elrad D, Grossman AR, Hippler M, Niyogi KK (2009) An ancient light-harvesting protein is critical for the regulation of algal photosynthesis. Nature 462:518-521

Pigolev AV, Klimov VV (2015) The green alga Chlamydomonas reinhardtii as a tool for in vivo study of site-directed mutations in PsbO protein of photosystem II. Biochemistry 80:785-797 (Moscow)

Pigolev AV, Zharmukhamedov SK, Klimov VV (2009) The PsbO mutant of Chlamydomonas reinhardtii is capable of assembling stable, photochemically active reaction center of photosystem II. Biol Membr 26:31-40

Pigolev AV, Timoshevsky DS, Klimov VV (2012) Effect of K223E and $\mathrm{K} 226 \mathrm{E}$ amino acid substitutions in $\mathrm{PsbO}$ protein of photosystem 2 on stability and functional activity of the wateroxidizing complex in Chlamydomonas reinhardtii. Biochemistry 77:71-77 (Moscow)

Rathner P, Mueller N, Wimmer R, Chandra K (2015) Solution NMR and molecular dynamics reveal a persistent alpha helix within the dynamic region of PsbQ from photosystem II of higher plants. Proteins 83:1677-1686

Roncel M, Kirilovsky D, Guerrero F, Serrano A, Ortega JM (2012) Photosynthetic cytochrome $c_{550}$. Biochim Biophys Acta 1817:1152-1163

Roose J, Wegener K, Pakrasi H (2007a) The extrinsic proteins of photosystem II. Photosyn Res 92:369-387

Roose JL, Kashino Y, Pakrasi HB (2007b) The PsbQ protein defines cyanobacterial Photosystem II complexes with highest activity and stability. Proc Natl Acad Sci USA 104:2548-2553

Roose JR, Frankel LK, Bricker TM (2010) Documentation of significant electron transport defects on the reducing side of photosystem II upon removal of the PsbP and PsbQ extrinsic proteins. Biochemistry 49:36-41

Schlodder E, Meyer B (1987) pH dependence of oxygen evolution and reduction kinetics of photooxidized chlorophyll aII (P-680) in photosystem II particles from Synechococcus sp. Biochim Biophys Acta BBA Bioenerg 890:23-31

Shen JR, Ikeuchi M, Inoue Y (1997) Analysis of the psbU gene encoding the $12-\mathrm{kDa}$ extrinsic protein of photosystem II and studies on its role by deletion mutagenesis in Synechocystis sp. PCC 6803. J Biol Chem 272:17821-17826

Shutova T, Klimov VV, Andersson B, Samuelsson G (2007) A cluster of carboxylic groups in PsbO protein is involved in proton transfer from the water oxidizing complex of photosystem II. Biochim Biophys Acta 1767:434-440
Summerfield TC, Crawford TS, Young RD, Chua JP, Macdonald RL, Sherman LA, Eaton-Rye JJ (2013) Environmental pH affects photoautotrophic growth of Synechocystis sp. PCC 6803 strainscarrying mutations in the lumenal proteins of PSII. Plant Cell Physiol 54:859-874

Summerfield TC, Winter RT, Eaton-Rye JJ (2005) Investigation of a requirement for the PsbP-like protein in Synechocystis sp. PCC 6803. Photosynth Res 84:263-268

Suorsa M, Aro E-M (2007) Expression, assembly, and auxiliary functions of photosystem II oxygen-evolving proteins in higher plants. Photosynth Res 93:89-100

Sveshnikov D, Funk C, Schroder WP (2007) The PsbP-like protein (sll1418) of Synechocystis sp. PCC 6803 stabilises the donor side of photosystem II. Photosynth Res 93:101-109

Takahashi M, Furuhashi T, Ishikawa N, Horiguchi G, Sakamoto A, Tsukaya H, Morikawa H (2014) Nitrogen dioxide regulates organ growth by controlling cell proliferation and enlargement in Arabidopsis. New Phytol 201:1304-1315

Takahashi M, Shigeto J, Sakamoto A, Izumi S, Asada K, Morikawa H (2015) Dual selective nitration in Arabidopsis: almost exclusive nitration of PsbO and PsbP, and highly susceptible nitration of four non PS II proteins, including peroxidoxin II E. Electrophoresis 36:2569-2578

Thornton LE, Ohkawa H, Roose JL, Kashino Y, Keren N, Pakrasi HB (2004) Homologs of plant PsbP and PsbQ proteins are necessary for regulation of photosystem II activity in the cyanobacterium Synechocystis 6803. Plant Cell 16:2164-2175

Tohri A, Dohmae N, Suzuki T, Ohta H, Inoue Y, Enami I (2004) Identification of domains on the extrinsic $23 \mathrm{kDa}$ protein possibly involved in electrostatic interaction with the extrinsic $33 \mathrm{kDa}$ protein in spinach photosystem II. Eur J Biochem 271:962-971

Tokutsu R, Minagawa J (2013) Energy-dissipative supercomplex of photosystem II associated with LHCSR3 in Chlamydomas reinhardtii. Proc Natl Acad Sci USA 110:10016-10021

Tomita M, Ifuku K, Sato F, Noguchi T (2009) FTIR evidence that the PsbP extrinsic protein induces protein conformational changes around the oxygen-evolving Mn cluster in photosystem II. Biochemistry 48:6318-6325

Trachootham D, Lu W, Ogasawara MA, Rivera-Del Valle N, Huang P (2015) Redox regulation of cell survival. Antioxid Redox Signal 10:1343-1347

Umena Y, Kawakami K, Shen JR, Kamiya N (2011) Crystal structure of oxygen-evolving photosystem II at a resolution of $1.9 \mathrm{~A}$. Nature 473:55-60

Uno C, Nagao R, Suzuki H, Tomo T, Noguchi T (2013) Structural coupling of extrinsic proteins with the oxygen-evolving center in red algal photosystem II as revealed by light-induced FTIR difference spectroscopy. Biochemistry 52:5705-5707

Vassiliev S, Zaraiskaya T, Bruce D (2012) Exploring the energetics of water permeation in photosystem II by multiple steered molecular dynamics simulations. Biochim Biophys Acta 1817:1671-1678

Vassiliev S, Zaraiskaya T, Bruce D (2013) Molecular dynamics simulations reveal highly permeable oxygen exit channels shared with water uptake channels in photosystem II. Biochim Biophys Acta 1827:1148-1155

Veerman J, Bentley FK, Eaton-Rye JJ, Mullineaux CW, Vasil'ev S, Bruce D (2005) The PsbU subunit of photosystem II stabilizes energy transfer and primary photochemistry in the phycobilisome-photosystem II assembly of Synechocystis sp. PCC 6803. Biochemistry 44:16939-16948

Vinyard DJ, Ananyev GM, Dismukes GC (2013) Photosystem II: the reaction center of oxygenic photosynthesis. Annu Rev Biochem 82:577-606 
Webber A, Packman LC, Gray JC (1989) A $10 \mathrm{kDa}$ polypeptide associated with the oxygen-evolving complex of photosystem II has a putative C-terminal noncleavable thylakoid transfer domain. FEBS Lett 242:435-438

Xue H, Tokutsu R, Bergner SV, Scholz M, Minagawa J, Hippler M (2015) Photosysten II subunit PsbR is required for efficient binding of light-harvesting complex stress-related protein 3 to photosystem II-light-harvesting supercomplexes in Chlamydomonas reinhardtii. Plant Physiol 167:1566-1578

Yano J, Yachandra V (2014) Mn4Ca cluster in photosynthesis: where and how water is oxidized to dioxygen. Chem Rev 114:4175-4205

Yi X, Hargett SR, Frankel LK, Bricker TM (2006) The PsbQ protein is required in Arabidopsis for photosystem II assembly/stability and photoautotrophy under low light conditions. J Biol Chem 281:26260-26267
Yi X, Liu H, Hargett SR, Frankel LK, Bricker TM (2007) The PsbP protein is required for photosystem II complex assembly/stability and photoautotrophy in Arabidopsis thaliana. J Biol Chem 282:24833-24841

Yi X, Hargett SR, Frankel LK, Bricker TM (2009) The PsbP protein, but not the PsbQ protein, is required for normal thylakoid membrane architecture in Arabidopsis thaliana. FEBS Lett 583:2142-2147

Young A, McChargue M, Frankel LK, Bricker TM, Putnam-Evans C (2002) Alterations of the oxygen-evolving apparatus induced by a 305Arg $\rightarrow$ 305Ser mutation in the CP43 protein of photosystem II from Synechocystis sp. PCC 6803 under chloride-limiting conditions. Biochemistry 41:15747-15753

Zhang YBB (2008) I-TASSER server for protein 3D structure prediction. BMC Bioinform 9:40-48 